\title{
Situierte Sicherheit. Für einen methodologischen Situationismus in den Critical Security Studies
}

Der Kopenhagener Schule ist immer wieder vorgeworfen worden, an der Idee einer ahistorischen Grammatik der Sicherheit festzuhalten, mit der in unterschiedlichen politischen Konstellationen unter bestimmten Bedingungen außergewöhnliche Maßnahmen zum Schutz von Referenzobjekten durchgesetzt werden können. Demgegenüber hat sich eine soziologische Lesart der Securitization Theory für ein stärker kontextualistisches Verständnis von Versicherheitlichung stark gemacht, ohne diesem aber eine konsistente, analytische Grundlage zu geben. Der Beitrag schlägt für eine solche Grundlage einen methodologischen Situationismus vor, der es erlaubt, den Blick stärker darauf zu richten, wie Sicherheit ein Bestandteil sozialer und politischer Wirkungszusammenhänge wird. Er knüpft dafür an Theorietraditionen des Pragmatismus und des Interaktionismus an, ergänzt diese aber um eine Perspektive auf transsituative Mechanismen, deren Funktionsweise am Beispiel von Forschungen zum security-development-nexus erläutert werden.

\section{Einleitung}

Obschon die Securitization Theory aus einer Kritik an der Verengung des Sicherheitsbegriffs auf zwischenstaatliche Beziehungen und militärisch verstandene Sicherheit hervorgegangen ist, mehrt sich inzwischen der Vorwurf eines traditionalistischen und essenzialistischen Sicherheitsverständnisses. ${ }^{1}$ Die jüngste Debatte um einen vermeintlichen Rassismus der Kopenhagener Schule trieb dieses Monitum noch einmal auf die Spitze, bezichtigen Alison Howell und Melanie Richter-Montpetit (2020) die Securitization Theory ${ }^{2}$ doch eines Sicherheitsverständnisses, das nicht nur rassistische Gewalt ignoriere, sondern den Widerstand gegen diese weitgehend delegitimiere, weil es am liberalen Status quo orientiert sei.

Auch wenn diese Kritik inzwischen nicht nur durch einige Autor*innen der Kopenhagener Schule zurückgewiesen wurde, die nicht zuletzt auf Unsauberkeiten in der Argumentation von Howell und Richter-Montpetit hinwiesen (Wæver/Buzan 2020; Hansen 2020), ist zumindest der Vorwurf des Traditionalismus, der hier freilich etwas schrill - als Kritik an der „methodological whiteness“ formuliert

1 Der Text ist im Rahmen konzeptioneller Diskussionen im DFG geförderten SFB/TRR 138 „Dynamiken der Sicherheit. Formen der Versicherheitlichung in historischer Perspektive“" entstanden. Ich danke vor allem Horst Carl, Angela Marciniak, Andreas Langenohl und Sven Opitz für viele hilfreiche Kommentare und Anregungen.

2 Der Begriff Securitization Theory soll hier anzeigen, dass sich über die Kopenhagener Schule hinaus ein breiterer theoretischer Diskussionszusammenhang rund um das Konzept der Versicherheitlichung etabliert hat, auf den der vorliegende Artikel Bezug nimmt. 
wird, nicht neu. So verweisen auch die beiden Autorinnen auf die Anfang der 2000er einsetzende feministische Diskussion um die Securitization Theory (Hansen 2000; Tickner 2004; Heck \& Schlag 2013), auf die Kritik am Eurozentrismus des Konzepts der normal politics (Bilgin 2010, 2011; Wilkinson 2007; Vuori 2008) sowie auf die demgegenüber jüngeren und an beide Debatten anschließenden Forderungen, die Bedeutung kolonialer Herrschaft auch für gegenwärtige Sicherheitspolitiken endlich ernst zu nehmen (Ketzmerick 2019).

Viele, wenngleich natürlich nicht alle Einwände, sind mit einer theoretischen Schwäche der Securitization Theory verbunden, die Gegenstand dieses Artikels ist und die auch bereits Anlass zu theoretischen Modifikationen und Weiterentwicklungen gegeben hat. In ihrer Kritik am realistischen Sicherheitsverständnis hat vor allem die Kopenhagener Schule darauf hingewiesen, dass Sicherheit nicht ein objektiver Zustand ist, sondern dass mit ihr eine spezifische Ordnungsleistung erbracht wird, durch die sich politische Konstellationen verändern. Dies geschieht vor allem dadurch, dass es Akteur*innen erfolgreich verstehen, Referenzobjekte als existenziell bedroht darzustellen und daraus die Notwendigkeit außergewöhnlicher politischer Entscheidungen abzuleiten. Sicherheit dient der Kopenhagener Schule zufolge gewissermaßen als kommunikatives Instrument der Herstellung bzw. des Wandels politischer Ordnung (Balzacq 2005: 171). Von Beginn an war, wie zuletzt Felix Ciută (2009) noch einmal gezeigt hat, die Securitization Theory damit zwischen zwei Annahmen hin- und hergerissen: der Annahme, die Ordnungsleistung von Sicherheit entspringe einer ahistorischen und universellen Logik von Sicherheit, einer „grammar of security“ (Buzan, Wæver/de Wilde 1998: 33) und der Annahme, dass diese Ordnungsleistung im Wesentlichen von den Akteur*innen geleistet wird, die jeweils definieren, was unter Sicherheit zu verstehen ist. Insbesondere die Annahme einer jede raum-zeitliche Situation transzendierenden Grammatik der Sicherheit läuft, wie vielfach bemerkt, auf ein essenzialistisches Verständnis von Sicherheit - freilich im Rahmen einer konstruktivistischen Methodologie - hinaus.

Der vorliegende Beitrag schlägt eine analytische Alternative zu diesem Essenzialismus vor, ohne den Boden der Securitization Theory gänzlich zu verlassen. Er geht dafür zurück zur ursprünglichen Intention der Securitization Theory, im Gegensatz zum objektivistischen Sicherheitsverständnis des Realismus die Frage nach der Ordnungs- und Strukturierungsleistung von Sicherheit in den Mittelpunkt zu stellen, knüpft dann aber an die soziologische Wende der Critical Security Studies $(\mathrm{CSS})^{3}$ an, deren Vertreter*innen vor allem auf die Relevanz kontextueller Faktoren für Versicherheitlichung hingewiesen haben (Balzacq 2005).

3 Ich verwende hier einen weiten Begriff von CSS. Die Bezeichnung wurde ursprünglich von Ken Booth (2011) für eine Sicherheitsforschung geprägt, die sich explizit an Grundsätzen der Kritischen Theorie orientiert (Schlegel/Schuck 2017). Demgegenüber hat sich mittlerweile durchgesetzt von verschiedenen Ansätzen und Generationen der CSS zu sprechen, die sich auf unterschiedliche Weise von realistischen Prämissen der Sicherheitsforschung abgrenzen (Peoples/Vaughan-Williams 2010). Gemeinsam ist ihnen, dass sie Sicherheit als Ergebnis intersubjektiver Deutungsprozesse betrachten. 
Allerdings bleibt die kontextualistische Lesart der Securitization Theory an einer entscheidenden Stelle analytisch unbefriedigend, denn es wurde bislang nicht geklärt, was - nicht zuletzt unter methodischen Gesichtspunkten - unter einem Kontext $\mathrm{zu}$ verstehen ist und wie sich die Grenzen eines Kontexts bestimmen lassen. Im Folgenden schlage ich vor, dieses Problem durch einen methodologischen Situationismus zu lösen, der an pragmatistische Ansätze anschließt und unter anderem in den Arbeiten von Karin Knorr-Cetina (1988) oder Adele Clarke (2005; Clarke et al. 2015) nicht zuletzt unter Bezugnahme auf die Actor-Network-Theory (ANT) von Bruno Latour entwickelt wurde. Im Kern argumentiert der methodologische Situationismus, dass Situationen raum-zeitlich identifizierbare Interaktionszusammenhänge sind, in denen soziale Ordnung erst generiert wird. Der analytische Schwerpunkt wird damit nicht auf eine erfolgreiche Anwendung einer Grammatik der Sicherheit gelegt, sondern darauf zu erfassen, wie Sicherheit erst zum Bestandteil einer raum-zeitlichen Situation wird. In der Literatur zur Securitization Theory ist Sicherheit in diesem Sinne immer wieder als situiert oder als situierte Aktivität beschrieben worden (Balzacq 2005; Bubandt 2005; Salter 2008; Stritzel 2011; Wæver 2000). Allerdings fehlt bislang eine analytische Grundlage dafür. Zur Ausarbeitung einer solchen möchte dieser Artikel einen Beitrag leisten. Mit diesem analytischen Zugang entkommt man zum einen dem Problem, von einer transsituativen Grammatik der Sicherheit auszugehen und damit in das Fahrwasser des Essenzialismus zu geraten. Zum anderen wird gegenüber einem weitgehend diffusen Kontextualismus eine methodische Rigidität gewonnen, da von konkreten Situationen ausgegangen wird, die gewissermaßen das Letztelement des Sozialen darstellen.

Im Folgenden werde ich zunächst noch einmal kurz auf die theoretische Ausgangslage der CSS und hier insbesondere der Kopenhagener Schule und der soziologischen Wende eingehen (2), bevor ich die Grundzüge des methodologischen Situationismus entfalte (3). Zwei offenkundige Schwierigkeiten ergeben sich indes mit einer situationsanalytischen Perspektive. Zum einen läuft sie Gefahr, die materielle Dimension von Situationen zu unterschätzen, indem sie einem Textualismus verhaftet bleibt, der dem Interaktionismus zu eigen ist (4). Zum anderen neigt die methodische Fokussierung auf die Situation zu einem gewissen Provinzialismus, wenn er Sicherheit ausschließlich als situierte Aktivität versteht. Das von Stefan Hirschauer (2014) entwickelte Konzept der Intersituativität verspricht hier insofern einen Ausweg, als es die Verbindung zwischen Situationen in den Mittelpunkt rückt und damit empirisch die Frage aufwirft, mit welchen Mechanismen übersituative Zusammenhänge hergestellt werden (5). Der hier unterbreitete Vorschlag knüpft an John Deweys Idee der Problemsituation an. Zum einen, so werde ich argumentieren, erlauben Heuristiken es Akteur*innen, Sicherheitsprobleme situativ zu identifizieren. Zum anderen greifen Akteur*innen in solchen Situationen dann auf Repertoires zurück, mit denen sie Sicherheitsprobleme bearbeiten. Heuristiken und Repertoires lassen sich somit als übersituative Mechanismen verstehen, mittels derer Akteur*innen Situationen verbinden, und die zugleich dazu beitragen, Bedeutungen von Sicherheit in neue Situationen zu übersetzen (Stritzel 2011). Die Funktionswei- 
se von Heuristiken und Repertoires erläutere ich schließlich unter Bezugnahme auf Forschungen zum sogenannten security-development-nexus (6).

Das Ziel des Artikels ist es, eine neue analytische Perspektive im Rahmen der CSS zu eröffnen, die die Probleme des Essenzialismus umgeht und den Kontextualismus analytisch anreichert. Dies soll im Wesentlichen auf dem Weg einer Diskussion theoretischer und analytischer Konzepte geschehen. Eine durchgängige, empirische Illustration und gar Anwendung des hier vorgeschlagenen methodologischen Situationismus muss daher anderen und späteren Arbeiten vorbehalten bleiben (aus historischer Perspektive etwa Kampmann/Carl 2021). Nichtsdestotrotz werde ich an verschiedenen Stellen versuchen, das konzeptionelle Argument empirisch zu plausibilisieren.

\section{Von der Historisierung von Sicherheit zur Versicherheitlichung}

Sucht man nach Ansätzen für ein situationsbezogenes Verständnis von Sicherheit, so wird man zunächst vor allem bei konzept- und begriffsgeschichtlichen Forschungen fündig, die auf die verschiedenen, historisch variablen Bedeutungsgehalte von Sicherheit hingewiesen und die Sicherheitsforschung damit für Analysen der Sicherheitssemantik selbst geöffnet haben (Rothschild 1995; Der Derian 1995; Neocleous 2006). Mit Niklas Luhmann (1990) könnte man sagen, dass damit von Waszu Wie-Fragen übergegangen wurde: Nicht, was Sicherheit ist, sondern wie Sicherheit in verschiedenen historischen Zusammenhängen verstanden wurde, geriet in den Blick. So hat Werner Conze (1984) begriffsgeschichtlich die politische Geschichte des Sicherheitsbegriffs rekonstruiert und gezeigt, dass sich die Bedeutung des Sicherheitsbegriffs in enger Abhängigkeit von gesellschaftlichen Entwicklungen - von der Entstehung des Fürstenstaats und seines Militärs über das europäische Staatensystem bis zur Gründung internationaler Institutionen und den Anfängen des Wohlfahrtsstaats - veränderte und differenzierte. ${ }^{4}$ Begriffs- und konzeptgeschichtliche Rekonstruktionen verweisen dabei durchgängig darauf, dass Sicherheit einerseits Wertbegriff geworden ist, der zum einen dazu dient, politische Ziele mit größerer Durchschlagskraft zu versehen und dessen Gehalt sich zum anderen abhängig von gesellschaftlichen Wertüberzeugungen wandelt (Kaufmann 1973). Christopher Daase (2010) hat dies an der Diskussion um die Erweiterung des Sicherheitsbegriffs nachvollzogen und argumentiert, dass in den Gesellschaften des Globalen Nordens in den 1970er und 1980er Jahren wirtschaftliche und ökologische, später dann auch humanitäre Aspekte in dem Maße Bestandteile von Sicherheitspolitik geworden sind, wie gesellschaftliche Diskurse neue Bedrohungspotenziale identifizierten. Die räumliche und sachliche Expansion des Sicherheitsbegriffs, die diskursive Entdeckung neuer Gefahren und die Ausweitung der Refe-

4 Baldwin (1997) hat demgegenüber die Konzeptanalyse dazu verwendet, einen Bedeutungskern von Sicherheit jenseits unterschiedlicher konzeptioneller Verständnisse zu rekonstruieren. 
renzobjekte von Sicherheit auf gesellschaftliche Gruppen und das Individuum markierten einen durchaus drastischen Bedeutungswandel von Sicherheit in der zweiten Hälfte des 20. Jahrhunderts gegenüber einem Sicherheitsverständnis, das lange Zeit auf Aspekte territorialer Verteidigung und der Schutzpflicht von Herrschenden gegenüber ihren Untertanen, also auf Fragen politischer Souveränität begrenzt war. Die amerikanische und französische Revolution verband den Sicherheitsbegriff hingegen bereits mit den natürlichen Menschenrechten, vor allem aber auch mit dem Prinzip der Volkssouveränität, so dass Sicherheit gleichbedeutend mit einer Garantie der Rechtsordnung wurde, die das Glück der Bürger*innen sicherstellen sollte (Conze 1984).

Nicht zuletzt unterstreicht dieses Beispiel die Relevanz einer historisierenden Perspektive auf die Bedeutungsgehalte des Sicherheitsbegriffs. Emma Rothschild (1995) hat dies in aller Klarheit in ihrem in vielerlei Hinsicht für eine historisierende Perspektive richtungsweisenden Artikel herausgearbeitet, in dem sie zeigt, dass Sicherheit, jedenfalls in der europäischen Tradition, im Kern das Verhältnis von Individuum auf der einen sowie Staat und Gesellschaft auf der anderen Seite betrifft. Mit anderen Worten: Die - im Sinne Luhmanns - alteuropäische Sicherheitssemantik fußte auf einer begrifflichen Unterscheidung von Individuum und Staat/Gesellschaft, die die Frage nach dem Verhältnis etwa von Freiheit und Sicherheit überhaupt erst möglich machte. Gesellschaftsstrukturell entsprach diese Unterscheidung der Dynamik funktionaler Differenzierung und der mit ihr verbundenen Notwendigkeit, das Verhältnis zwischen den Steuerungsambitionen staatlicher Politik und anderen gesellschaftlichen Teilbereichen neu zu tarieren. Die Erweiterung der Bedeutung von Sicherheit um das Referenzobjekt des Individuums - etwa im Rahmen des Konzepts der human security in den 1990er Jahren - greift in dieser Hinsicht die liberale Idee individueller Sicherheit auf, so dass Rothschild schlussfolgert: „The new security principles of the end of the twentieth century constitute a rediscovery, of sorts, of this late eighteenth and early nineteenth-century politic“ (Rothschild 1995: 65).

Die Historisierung von Sicherheit im Sinne einer Rekonstruktion sich kontextuell wandelnder Bedeutungsgehalte fördert auf diese Weise Kontinuitäten und Diskontinuitäten der Verwendungsweisen von Sicherheit als Teil des politischen Vokabulars zutage. Der Wandel und die Reinterpretation der Bedeutung von Sicherheit ist dabei, Rothschild zufolge, eng mit gesellschaftlichen Krisenerfahrungen verbunden von den napoleonischen und revolutionären Kriegen sowie den beiden Weltkriegen bis hin zu den osteuropäischen Revolutionen von 1989. Im Zusammenhang solcher Krisen erscheinen, so ließe sich schlussfolgern, vorhandene Deutungsmuster von Sicherheit und mit ihnen verbundene Handlungsmöglichkeiten zunehmend unplausibel. Situativ entstehen dann neue Bedeutungen oder Bedeutungen werden, wie das Beispiel der menschlichen Sicherheit zeigt, re-interpretiert und modifiziert.

Vor dem Hintergrund einer solchen historisierenden Betrachtungsweise lassen sich auch die CSS und insbesondere die Securitization Theory historisch einordnen. In dem Maße wie (nationale) Sicherheit seit den 1940er Jahren, zunächst in den USA, dann weit darüber hinaus, zum raison d'état wurde, konnten mit Verweis auf 
existenzielle Bedrohungen außergewöhnliche Maßnahmen legitimiert werden, die dem Staat neue Eingriffs- und Regulierungsmöglichkeiten eröffneten und die Kompetenzen der Exekutive ausweiteten (Wæver 2008: 102). Die Kopenhagener Schule hat dies zum Anlass genommen, nach den Mechanismen, Bedingungen und Folgen einer solchen Versicherheitlichung zu fragen, durch die demokratische Prozesse gleichsam von innen ausgehöhlt und internationale wie gesellschaftliche Konflikte verschärft werden. Auch das von Barry Buzan und Ole Wæver (Wæver 1995) entwickelte Konzept der desecuritization lässt sich in diesem Zusammenhang insofern historisieren, als es darauf zielte, Diplomatie und politische Verhandlungen als konstruktive und deeskalierende Alternative zu einer Sicherheitslogik erscheinen zu lassen, die den Ost-West-Konflikt in den 1980er Jahren prägte (Guzzini 2015).

Zugleich markieren die CSS damit aber auch einen Wechsel in der Fragestellung, die weg von der begriffs- und konzeptgeschichtlichen Rekonstruktion hin zur empirischen Analyse der sozialen Konstruktion von Sicherheit führt. ${ }^{5}$ Gut nachvollziehbar wird dieser Wechsel in Buzans „People, States and Fear“ (Buzan 1983), das unter Verweis auf William Gallie (1956) - mit der Feststellung beginnt, Sicherheit sei ein „essentially contested concept“, dann aber darin mündet, unterschiedliche Bedeutungen von Sicherheit in Abhängigkeit von Kenneth Waltz' „levels of analysis" herauszuarbeiten (Waltz 1959). Sicherheit erscheint damit als ein komplexes und im Kern - dank der auf den unterschiedlichen Ebenen virulenten Sicherheitsbedürfnisse - auch widersprüchliches Konzept. Gleichwohl bleibt „People, States and Fear" noch desinteressiert an der empirischen und politisch folgenreichen Konstruktion von Bedrohungen. Diese gerät dann erst mit einem „kritischen Konstruktivismus" (Buzan/Hansen 2009: 197) in den Blick, der davon ausgeht, dass Sicherheit „emerges and changes as a result of discourses and discursive actions intended to reproduce historical structures and subjects within states and among them" (Lipschutz 1995: 17). $\mathrm{Zu}$ diesem kritischen Konstruktivismus gehören unter anderem Arbeiten, die sich der diskursiven Konstruktion von Unsicherheit und Bedrohungen widmen (u.a. Campbell 1992; Weldes et al. 1999). Parallel entstehen aus der institutionalistischen Tradition der Internationalen Beziehungen (IB) heraus Ansätze, die Konstruktionen nationaler Sicherheitskultur mit den normativen Erwartungen ihrer internationalen Umwelt in Verbindung bringen (vor allem Katzenstein 1996).

Diese Entwicklung von einer historisierenden zu einer sozial-konstruktivistischen Analyse der Konstruktion von Bedrohung und ihrer Wirkung ${ }^{6}$ in verschiedenen politischen Konstellationen führt schließlich in das Forschungsprogramm der Kopenhagener Schule, die den semantischen Gehalt von Sicherheit in sozialen Verwendungszusammenhängen in den Mittelpunkt rücken: „In this approach, the meaning

5 Eine gänzlich andere Richtung einer Historisierung von Sicherheit schlagen Arbeiten im Anschluss an Michel Foucault ein, die gegenüber begriffsgeschichtlichen Perspektiven an der Genealogie gesellschaftlicher Problematisierungen durch Sicherheit ansetzen. Im Mittelpunkt steht hier die Frage, wie die liberale Vorstellung von Sicherheit konstruktivistisch erst eine staatliche Interventionspraxis hervorgebracht hat (vgl. etwa die Beiträge in Dillon/ Neal 2008).

6 Vgl. zu den unterschiedlichen Verständnissen von Wirkung als Zusammenhang zwischen Legimitation und Effektivität von Versicherheitlichung Andreas Langenohl 2019. 
of a concept lies in its usage and is not something we can define analytically or philosophically according to what would be ,best"“ (Buzan, Wæver/de Wilde 1998: 24). Damit ist der Schritt von der Konzeptgeschichte hin zur Analyse von Sprechakten und Diskursen gemacht.

\section{Die soziologische Wende der CSS}

Zwei Aspekte markieren das besondere Erkenntnisinteresse der Securitization Theory wie es von der Kopenhagener Schule zunächst formuliert wurde: Zum einen fokussiert sie die kommunikative Struktur des Versicherheitlichungsprozesses und versucht, die Grammatik von Sicherheit zu entschlüsseln. Dahinter steht letztlich die Vermutung, dass die besondere Sprechweise des securitizing move gewissermaßen unter Absehung besonderer Umstände stets zum gleichen Ergebnis führt, nämlich zum Bruch mit den Regeln normaler Politik (Wæver 1995). In „Security - A new framework for analysis“ wird diese Vorstellung bezeichnenderweise mit dem metaphorischen Bild von Grammatik und Dialekt eingeführt: Die Grammatik der Sicherheit existiert unterhalb ihrer verschiedenen Dialekte, die in den unterschiedlichen - militärischen, ökonomischen, gesellschaftlichen, politischen und Umweltsektoren der Sicherheit gesprochen werden (Buzan, Wæver/de Wilde 1998: 33). Zum anderen interessiert sich die Securitization Theory für die politischen Wirkungen einer erfolgreichen Anwendung dieser Grammatik in Sprechakten im Sinne einer gelungenen Versicherheitlichung.

Zweifelsohne hat die Securitization Theory damit einen wichtigen Grundstein für die Analyse der politischen Folgen der diskursiven Verknüpfung von Sicherheit mit bestimmten Themen gelegt. Dennoch haben vor allem drei Kritiken dazu geführt, dass sich in Abgrenzung von der Annahme einer transsituativen Grammatik der Sicherheit eine alternative Lesart von Versicherheitlichung entwickelte.

Die erste Kritik betrifft die Rolle des Publikums, das zwar als ein konstitutiver Bestandteil der intersubjektiven Generierung von Bedrohungskonstruktionen erscheint, selbst aber merkwürdig passiv bleibt. Demgegenüber haben verschiedene Autorinnen und Autoren vorgeschlagen, das Publikum selbst als Akteur aufzufassen und die ursprüngliche Vorstellung einer intersubjektiven Einigung über existenzielle Bedrohungen gewissermaßen als interaktive Akteurskonstellation und nicht im Sinne eines Sender-Empfänger-Modells von Kommunikation zwischen dem securitizing actor und einem mehr oder weniger diffusen Kollektiv zu verstehen (Côté 2016; Leonard/Kaunert 2011). Adam Côté (2016: 550) radikalisiert diese Sichtweise bis zu dem Punkt, dass securitzing actor und Publikum letztlich als Akteur*innen konzipiert werden, die sich über ihre verschiedenen Wahrnehmungen von Sicherheit austauschen. Obschon diese Auffassung externe Bedingungen und Restriktionen sicherheitsbezogener Interaktionen ausblendet, verweist sie doch darauf, dass die These einer intersubjektiven Aushandlung der Bedeutung von Sicherheit letztlich einer etwas anspruchsvolleren analytischen Konzeption von Interaktion bedarf, die in der ursprünglichen Fassung der Securitization Theory fehlt. 
Die zweite Kritik bezieht sich auf die enge Bindung von Sicherheit an den Sprechakt selbst, durch den existenzielle Bedrohungen zu allererst als solche erscheinen. Insbesondere Lene Hansen (2000) hat hervorgehoben, dass unter dieser Prämisse aus dem Blick gerät, dass bestimmte Akteur*innen ihre Interpretationen von Sicherheit und Bedrohung überhaupt nicht artikulieren können. In die gleiche Richtung weist die jüngere postkoloniale Kritik, die unter anderem bezweifelt, dass sich mit den analytischen Mitteln der Securitization Theory die Exklusion der Subalternen aus einem öffentlichen Sicherheitsdiskurs analysieren lässt (Bertrand 2018; Howell/Richter-Montpetit 2020). Hansen deutete allerdings schon früh in der Diskussion eine interessante Richtung an, wie sich eine Nichtartikulation von Sicherheit analytisch in den Blick nehmen lässt. „Security as silence "entsteht ihr zufolge, wenn Bedrohungen nicht benannt werden können, d.h. wenn es für Akteur*innen unmöglich oder sogar gefährlich ist, die von ihnen erfahrene Unsicherheit - und deren Verursacher*innen - öffentlich zu benennen (Hansen 2000: 287). Anstatt nun nur auf die Praktiken der Bedeutungszuschreibung zu fokussieren, müsse, so Hansen, die Situation selbst in den Blick genommen werden, in der Sprechakte artikuliert werden, denn ,security as silence“ ist nichts anderes als ,, situation where the potential subject of security has no, or limited, possibility of speaking its security problem" (Hansen 2000: 287). Hansens Kritik an der Sprechaktfixierung der Securitization Theory lässt sich hier als erster wichtiger Hinweis verstehen, dass Sprechakte in konkreten Situationen entstehen, deren Analyse die Einbeziehung nichtkommunikativer Elemente erfordert.

Die dritte Kritik problematisiert das Spannungsverhältnis von Essenzialismus und Konstruktivismus in der Securitization Theory. Während Ciută zufolge die Securitization Theory auf der einen Seite behauptet, ,security is what actors make of it“" (Buzan \& Wæver 2003: 48; zitiert nach Ciută 2009: 302), hält sie auf der anderen Seite an einem Bedeutungskern von Sicherheit fest. So formulieren Buzan et al. unmissverständlich „security is about survival“ (Buzan, Wæver/de Wilde 1998: 21), um dann aber die Bedeutungen von Sicherheit den Definitionspraktiken der Akteur*innen zu überlassen. Dass dies für die Analyse von Prozessen der Versicherheitlichung nicht weiter ins Gewicht fällt, hat damit zu tun, dass sich die Securitization Theory letztlich nicht mehr für die Bedeutungen von Sicherheit interessiert, sondern für die Konstruktion existenzieller Bedrohungen von Referenzobjekten. Die Frage nach sich wandelnden und miteinander in Konflikt geratenen Bedeutungen von Sicherheit, die noch im Mittelpunkt der historisierenden begriffs- und konzeptgeschichtlichen Rekonstruktion standen, rückt hier zugunsten der Analyse von Bedrohungskommunikation in den Hintergrund. Paradoxerweise führt dies dazu, so Ciută (2009: 310), dass eine der wichtigsten Theorien der CSS, Sicherheit selbst aus dem analytischen Vokabular der CSS ausklammert. Im Gegensatz dazu müsse, so die Schlussfolgerung Ciutăs, die CSS eine hermeneutische Perspektive einnehmen, um sich wieder der sinnhaften Konstruktion von Sicherheit - und nicht nur von Bedrohungen - zuzuwenden.

Alle drei Kritiken münden schließlich in stärker kontextualistische Lesarten von Versicherheitlichung, wie sie vor allem von der soziologischen Wende der Securi- 
tization Theory vorangetrieben wurden (Stritzel 2007; McDonald 2008; Balzacq 2011; Klüfers 2014; Stritzel 2014; Philipsen 2020) und in der empirischen Forschung Bestätigung gefunden haben (Lucke, Diez/Wellmann 2016; Balzacq 2015).

In ihren unterschiedlichen Facetten laufen diese Lesarten darauf hinaus, die gesellschaftlichen, politischen und kulturellen Kontexte in den Mittelpunkt zu stellen, in denen Bedrohungen konstruiert werden. Pate stehen dabei verschiedene soziologische Ansätze wie etwa der symbolische Interaktionismus (Balzacq 2002) oder Pierre Bourdieus Feldtheorie (Bigo 2008). Nicht zuletzt die Arbeiten von Didier Bigo und der an ihn anschließenden Internationalen Politischen Soziologie der CSS (Burgess 2017), aber auch neuere Beiträge der Science \& Technology Studies haben die Bedeutung kontextueller Spezifika hervorgehoben, die für die Entstehung und Modifikation sicherheitsbezogener Praktiken etwa der Überwachung und Kontrolle entscheidend sind. Dazu zählen nicht zuletzt technologische Innovationen (Bellanova, Jacobsen/Monsees 2020) oder die Entstehung bestimmter Berufsgruppen (Petersen 2013). ${ }^{7}$

Am konsequentesten hat Thierry Balzacq den Kontextualismus theoretisch ausformuliert. Dabei knüpft er an die aus der phänomenologischen Soziologie stammende These an, dass Sprechakte nur verstanden werden können, wenn Sprecher*innen und Publikum über den gleichen Bedeutungshorizont verfügen, mithin eine gemeinsame Lebenswelt teilen (Schütz/Luckmann 1979). Erst dann könnten securitizing moves eine Plausibilität für sich beanspruchen und auf Zustimmung hoffen. Er wendet sich damit explizit von der Vorstellung ab, Versicherheitlichung sei ein Resultat des illokutionären Sprechakts, der das hervorbringt, was er benennt, und knüpft demgegenüber an die Auffassung an, Versicherheitlichung stelle sich als Folge eines perlokutionären Sprechakts ein, also etwa als Zustimmung von Akteur*innen zum securitizing move eines anderen. Der Kontext bildet damit eine gesellschaftliche Bedingung der Möglichkeit einer erfolgreichen Versicherheitlichung und wird nicht - wie in der Vorstellung einer kontextübergreifenden Grammatik der Sicherheit (Balzacq 2005: 180) - vom Sicherheitssprechakt erst hervorgebracht. Diese kontextualistische Lesart von Versicherheitlichung führt schließlich zu erstaunlich subjektivistischen Schlussfolgerungen. So knüpft Balzacq (2005:192) die Bedingung einer erfolgreichen Versicherheitlichung etwa an die Bereitschaft eines Publikums, von einem securitizing move überzeugt zu werden und an die Kapazität des securitizing actors ein räsonierendes Vokabular zu finden, das das Publikum in geeigneter Weise anspricht.

Zwar mag der Kontextualismus, der auch von Ciută und Hansen als Lösung der von ihnen aufgeworfenen Probleme der Securitization Theory in Anschlag gebracht wird, auf den ersten Blick eher überzeugen als die Annahme einer kontextlosen Grammatik der Sicherheit. Indes bleibt in nahezu allen Versuchen, eine kontextua-

7 Zahlreiche empirische Arbeiten, die in diesem Zusammenhang in den letzten Jahren entstanden sind, verweisen auf die Vielzahl solcher kontextueller Faktoren, ohne dass sich daraus eine konsistente analytische Perspektive entwickelt hätte. Charakteristisch ist eher ein loses Anknüpfen an verschiedene Gesellschafts- und Sozialtheorien von Foucault bis Latour. 
listische Interpretation von Versicherheitlichung zu etablieren, der Begriff des Kontexts selbst merkwürdig unbestimmt. Das mag, wie die dekonstruktive Kritik am kontextualistischen Bedeutungsbegriff nahelegt (Derrida 1990), grundsätzlicher methodologischer Probleme geschuldet sein. Aber auch unabhängig davon müsste eine kontextualistische Theorie der Versicherheitlichung über Kriterien verfügen, um die Grenzen eines Kontexts zu bestimmen. Die im Folgenden vorgeschlagene Perspektive des methodologischen Situationismus versucht deshalb, dem Kontextualismus eine größere analytische Tiefenschärfe zu geben.

\section{Grundzüge des methodologischen Situationismus}

Im Mittelpunkt der theoretischen wie empirischen Arbeiten der securitziation studies stehen die Wirkungen, die die Konstruktion existenzieller Bedrohungen auf politische Prozesse haben. Diese reichen typischerweise von der Abkürzung politischer Deliberation unter Verweis auf die Dringlichkeit des Entscheidens und einer generellen Beschleunigung politischen Handels bis zur Umsetzung außergewöhnlicher Maßnahmen. Will man die Aufmerksamkeit nun weg von der Wirkung von Bedrohungskonstruktionen hin zur Herstellung eines Wirkungszusammenhangs von Sicherheit richten, bietet es sich an, am Pragmatismus anzusetzen. Die Arbeiten von John Dewey (1938) und George Mead (1968) - die dann später vor allem durch die Rezeption Herbert Blumers (1986) die Grundlage für ein symbolisch-interaktionistisches Verständnis situativer Ordnungsgenese wurden - eint, dass sie an der Herstellung gemeinsamer Handlungszusammenhänge interessiert sind, statt jene als gegeben vorauszusetzen. Während allerdings der Interaktionismus dazu neigt, die Situation strikt als physische Ko-Präsenz, also als Anwesenheit von Individuen aufzufassen (Gofman 1972), erlaubt der Pragmatismus eine Perspektive darauf, wie Anwesenheit auch ohne physische Präsenz hergestellt werden kann, so dass auch physisch Abwesendes - sowohl abwesende Individuen oder Kollektive als auch Objekte, Territorien oder nicht unmittelbar sinnlich Wahrnehmbares wie Bakterien oder Viren - Bestandteil einer Situation werden kann. Techniken der Herstellung von Anwesenheit sind in diesem Zusammenhang etwa Karten - beispielsweise mit eingezeichneten Sicherheitszonen (Lemay-Hébert 2018) -, digitale Kommunikationsformate, aber auch Apparate wie Mikroskope oder Scanner sowie technische Simulationen, die es ermöglichen, Situationen gleichsam vollständig aus einem lokalen Bezug zu entbetten und, wie Sven Opitz (2017) am Beispiel der Simulation von Pandemien gezeigt hat, als globale Situation zu konstituieren.

Für die CSS bietet es sich daher an, auf das ursprüngliche pragmatistische Verständnis zurückzugehen, weil dieses erlaubt, einen genaueren Blick auf die situative Konstitution von Sicherheit mit Hilfe solcher Techniken zu werfen. ${ }^{8}$ Ein pragmatistisches Verständnis von Sicherheit teilt mit dem Konstruktivismus die Annahme,

8 Auch in den Internationalen Beziehungen erfreuen sich sowohl Pragmatismus als auch daran anschließende Praxistheorien seit einiger Zeit einer steigenden Beliebtheit. Für einen 
dass Sicherheit einen relationalen Charakter besitzt, d.h. dass sich erst in sozialen Handlungszusammenhängen herauskristallisiert, was unter Sicherheit verstanden wird. Gegenüber dem Konstruktivismus beharrt er indes darauf, dass sich die Bedeutung von Sicherheit nicht in intersubjektiven Sinnzuschreibungen, etwa im Rahmen gemeinsam geteilter Bedrohungswahrnehmungen, erschöpft. Vielmehr legt der Pragmatismus einen Schwerpunkt auf den Handlungsvollzug selbst. Versteht man Handeln grundsätzlich als eine wissensbasierte Tätigkeit, dann beruht Wissen für den Pragmatismus nicht zuletzt auf einem know how, d.h. auf einem praktischen Können und damit vor allem auf einem impliziten Wissen (Reckwitz 2003: 289). Vor allem Dewey hat Handeln als eine Art kontinuierliches Problemlösen beschrieben, das sich im Wesentlichen in Routinen vollzieht und nur bei Irritationen und in der problematischen Situation, wenn sich also Probleme nicht mehr gleichsam automatisch lösen lassen, reflexiv wird (Pettenkofer 2017). In den CSS ist diese Betonung vorreflexiven Handelns vor allem von der sogenannten Pariser Schule in den Mittelpunkt gerückt worden, um deutlich zu machen, dass Versicherheitlichung nicht zwingend durch besondere Sprechakte hervorgebracht werden muss, sondern sich im Wesentlichen im normalen Alltagshandeln einstellt (Huysmans 2011).

Dewey hat damit auch den Grundstein für einen theoretisch gehaltvollen Begriff der Situation gelegt, indem er betont, dass Bedeutungen, die unseren Urteilen zugrunde liegen, in Situationen entstehen:

„(...) what is designated by the word 'situation' is not a single object or event or set of objects and events. For we never experience nor form judgments about objects and events in isolation, but only in connection with a contextual whole. The latter is what is called a "situation"“ (Dewey 1938: 66).

Insofern lassen sich Situationen letztlich auch nicht als isolierte Einheiten betrachten, sondern als „little systems of organized activities“ (Knorr-Cetina 1988: 29), die eine Vielfalt von Bezügen aufweisen. Situationen mögen, mit anderen Worten, den Vorteil besitzen, den unendlichen Strom des Sozialen gewissermaßen dingfest zu machen. Methodisch dienen sie aber vor allem als Ausgangspunkt für eine umfassendere Analyse all dessen, was konstitutiv für eine Situation ist. Karin Knorr-Cetina hat vor diesem Hintergrund die Tradition eines methodologischen Situationismus rekonstruiert, die in der Soziologie, so Knorr-Cetina, spätestens mit Georg Simmel anfängt. Diese Tradition schreibt pragmatistische Vorstellungen in zweifacher Hinsicht fort. Zum einen geht sie davon aus, dass menschliches Handeln immer in Situationen stattfindet. Zum anderen enthält sie aber ein von KnorrCetina in den Mittelpunkt gerücktes methodologisches Prinzip:

„I shall call methodological situationalism the principle which demands that descriptively adequate accounts of large-scale social phenomena be grounded in statements about actual social behaviour in concrete situations" (Knorr-Cetina 1988: 22).

In zweifacher Hinsicht verfährt der methodologische Situationismus Knorr-Cetina zufolge reduktionistisch. Erstens nimmt er an, dass makrosoziale Einheiten wie

Überblick siehe Hellmann 2017; Bueger/Gadinger 2008; Adler/Pouliot 2011. Zu Praxistheorien in den Security Studies siehe Bueger 2018. 
der Staat letztlich auf mikrosozialen Interaktionen beispielsweise zwischen Regierungsbeamt*innen beruhen. Dies ist auf den ersten Blick durchaus kompatibel mit der Forderung der Kopenhagener Schule, Versicherheitlichung an konkreten Sprechakten nachzuvollziehen. Allerdings legt Deweys Verständnis der Situation nahe, eine Situationsanalyse einerseits nicht nur auf Sprechakte zu konzentrieren und andererseits das Publikum als einen Bestandteil der Situation und damit der konkreten Interaktion zu verstehen. Zweitens, so Knorr-Cetina, lassen sich qualitative oder quantitative Daten über Makrophänomene selbst auf Praktiken zurückführen, die in bestimmten sozialen Situationen entstanden sind. Damit geraten performative Praktiken jenseits von Sprechakten in den Blick, mit denen Sicherheit als intelligibles Phänomen konstituiert wird - etwa durch Statistiken, Visualisierungen, oder die Programmierung von Algorithmen (Amoore 2013).

Der methodologische Situationismus trennt folglich nicht zwischen Sprechakt und Kontext, sondern nimmt die soziale Konstitution eines Wirkungszusammenhangs in den Blick, in dem Sicherheit dann politische Folgen entfaltet. So argumentiert etwa Andreas Langenohl (2020: 14), dass Versicherheitlichung im Kern darin besteht, eine sicherheitsbezogene Situationsdefinition herzustellen, die für Akteur*innen und Institutionen einen Unterschied macht und von ihnen nicht länger ignoriert werden kann. Die Situationsanalyse interessiert sich, mit anderen Worten, nicht so sehr für die Versicherheitlichung als Effekt einer Bedrohungskonstruktion, sondern dafür, wie es überhaupt dazu kommt, dass Sicherheit in einer bestimmten Situation vielfältige Effekte entfalten kann. Damit stellt sich nicht das Problem, wo die Grenze zwischen beobachtbarem Sprechakt und unsichtbarem Kontext verläuft. Vielmehr dient die Situation selbst als Ausgangspunkt der Analyse. Ihr kommt, in den Worten Knorr-Cetinas (1988: 31), eine ,analytical primacy“ in der Analyse sozialer Ordnung zu, weil sie präzisere Erklärungsressourcen bietet als ein vager Rückgriff auf kontextuelle Randbedingungen (Hirschauer 2014: 110; Schegloff 1997). Wer also etwa die Ergänzung der Bedeutung von Sicherheit durch neue Referenzobjekte wie dem Individuum oder der Umwelt empirisch nachvollziehen will, sollte dem methodologischen Situationismus zufolge nicht mit der Analyse von Makrodiskursen beginnen, sondern von einer lokalisierbaren Situation - etwa der Umsetzung eines Projekts der Entwicklungszusammenarbeit zur Stärkung menschlicher Sicherheit - ausgehen, in der solche Referenzobjekte konstruiert werden. Von dort lassen sich dann Interaktionszusammenhänge erschließen, die für eine solche Konstruktion konstitutiv sind - beispielsweise neben den einschlägigen Diskursen zu Sicherheit und Entwicklung auch die statistische Erfassung menschlicher Unsicherheit, die Festlegung von Projektzielen oder die Interaktionen zwischen den „drei Arenen“ der Intervention (Schlichte/Veit 2010), also der Zentrale, dem nationalen Büro und dem Ort der Projektdurchführung, in denen vermutlich durchaus unterschiedliche Bedeutungen von Sicherheit mobilisiert werden. Es ist gerade dieses Zusammenwirken unterschiedlicher Aktivitäten, das im methodologischen Situationismus in den Blick gerät, der nicht zwischen Kontext und Versicherheitlichung trennt, sondern Versicherheitlichung letztlich als Zusammenspiel unterschiedlicher, aber empirisch miteinander verbundener Faktoren versteht. 
Wie bereits angedeutet, enthält die Kritik an dem Verständnis von Sicherheit als illokutionärem Sprechakt bereits Hinweise auf die Fruchtbarkeit einer situationsanalytischen Reformulierung des Kontextualismus in den CSS. So fasst Adam Côté (2016) das Verhältnis von securitzing actor und Publikum als situative Aushandlung von Bedrohungswahrnehmungen. Ciutǎ (2009) plädiert dafür, sich den empirischen Deutungspraktiken von Akteur*innen zuzuwenden. Und Hansen hat in ihrer Verteidigung gegen die jüngste Kritik von Howell und Richter-Montpetit ebenfalls in die Richtung eines situationistischen Verständnisses von Sicherheit argumentiert, indem sie auf die konkrete Situation als Ausgangspunkt für eine Theoretisierung verweist, statt umgekehrt theoretische Konzepte der empirischen Analyse vorauszusetzen. Gerade eine gegenüber konkreten empirischen Situationen immune Sprechakttheorie verfehlt Hansen zufolge die besonderen Bedingungen der Situation, in der sich die Bedeutung von Sicherheit konstituiert. Sie zieht ihre Schlussfolgerung aus der Untersuchung der Art und Weise, wie die Subjektivität von Frauen und Mädchen in pakistanischen Rechtsdokumenten auf eine Weise konstituiert wird, die Ehrenmorde nicht als gesellschaftliches Sicherheitsproblem erscheinen lassen, sondern als etwas, das im Wesentlichen durch das Verhalten der Frauen selbst verursacht wurde.

An diesem Beispiel macht sie zweierlei deutlich: Erstens müssen für eine Analyse der Situation mehr als nur Sprechakte einbezogen werden, nämlich die Bedingungen dafür, dass jemand als anwesend in der Situation behandelt wird. Die von ihr in den Mittelpunkt gerückten Strategien des silencing, also des Ausschließens der Möglichkeit, Bedrohungserfahrungen zu artikulieren, lassen sich situationsanalytisch als Praktiken verstehen, Anwesenheit so herzustellen, dass bestimmte Akteur*innen von vornherein abwesend sind und es auch bleiben. Obgleich die existenzielle Bedrohung von Frauen eigentlich ein öffentliches Sicherheitsproblem darstellen müsste, führt ihre Behandlung in öffentlichen und vor allem auch in Rechtsdiskursen dazu, dass sie nicht als Akteur*innen in Erscheinung treten, weil ihnen gewissermaßen der Akteur*innenstatus genommen wurde. In diesem Zusammenhang weist Hansen auch auf die Bedeutung des Körpers für ein umfassendes Verständnis der Bedeutungskonstitution von Sicherheit hin. Sie wählt dafür das Bild der Kleinen Meerjungfrau: ,The tale of the Little Mermaid highlights the importance of voice and body for the construction of subjectivity, and it speaks about the chances, even deadly ones, one might take in the pursuit of desire and happiness“" (Hansen 2000: 285). Der Körper sei aber, so Hansen unter Rekurs auf Judith Butler (vgl. Butler 1993), nicht einfach gegeben, sondern selbst Teil eines Prozesses der diskursiven Herstellung von Bedeutung. Situationsanalytisch macht ihre Rekonstruktion der Rechts- und politischen Diskurse deutlich, dass der Körper allein noch keine Anwesenheit und damit eine situative Handlungsmöglichkeit garantiert, sondern dass diese durchaus voraussetzungsvoll ist. Indem, wie Hansen zeigt, der weibliche Körper in den entsprechenden Diskursen als ein individueller behandelt wird, erscheinen die mit Ehrenmorden verbundenen Sicherheitsprobleme nicht als öffentliche und damit als kollektive Probleme. Sie werden, mit anderen Worten, nicht Bestandteil einer Situation. 
Zweitens sind Situationen auf eine bestimmte Weise so strukturiert, dass in ihnen Verantwortlichkeiten und Rollen von Akteur*innen definiert werden. Die von Hansen untersuchten Dokumente und Praktiken definieren die Situation des Ehrenmordes weitgehend als eine private Angelegenheit:

„(...) the situation can be described as one where one group of private actors ('women') are being abused by another group of private actors, but where the state fails, or refuses to protect the formers security“" (Hansen 2000: 293).

Rekonstruiert man also die Bedeutung von Sicherheit aus der Situation heraus, dann wird in diesem Fall deutlich, dass nicht nur Sprechakte Bedeutungen von Sicherheit festlegen, sondern dass in der Situation selbst Bedingungen darüber zu finden sind, wer überhaupt sprechen kann und welche Bedeutungen artikulierbar sind.

Hansens Untersuchung eignet sich schließlich auch dafür, deutlich zu machen, was als Situation gilt. Die Praxis des Ehrenmordes als sicherheitsbezogene Situation zu analysieren heißt, Sicherheit - und vor allem auch ihre Abwesenheit - in der Situation selbst zu rekonstruieren. Die Gewaltpraxis wird erst möglich, weil die physische Sicherheit der Mädchen und Frauen in dieser Situation keine Rolle spielt. Genau deshalb plädiert Hansen dafür, dass die CSS das silencing stärker in den Blick nehmen sollten. Dies erfordert allerdings eine methodische Neuorientierung, die weg von der Sprechakttheorie und hin zu einer Berücksichtigung situativer Bedingungen der Produktion von Sicherheit und Unsicherheit führt.

Zugleich offenbart Hansens Analyse auch zwei Probleme des Situationismus, die es im Folgenden zu adressieren gilt. Zum einen neigt der Situationismus zu einem gewissen Provinzialismus, in dem Versicherheitlichung exklusiv aus der Situation her analysiert wird. Bei allen Vorteilen, die dies für eine empirische Untersuchung und eine darauf beruhende Generierung von Theorie haben mag, läuft die Beschränkung auf die Situation Gefahr, wichtige Bedingungen - hier etwa Fragen nach dem pakistanischen Rechtssystem oder einer patriarchalen Tradition - außer Acht zu lassen. Tatsächlich hat Hansen nicht die Praxis des Ehrenmordes, sondern vor allem die der Situation des Ehrenmordes vorausgehenden Bedingungen der Bedeutungsgenese von Sicherheit untersucht. Diese werden dann in der Situation selbst gewissermaßen aktualisiert. Methodisch interessant ist an Hansens Studie vor allem, dass sie diese Bedeutungsgenese von einer bestimmten Situation ausgehend rekonstruiert, die sich zudem gerade nicht durch eine erfolgreiche Versicherheitlichung im Sinne der Securitization Theory auszeichnet.

Zum anderen läuft der Situationismus auf den ersten Blick Gefahr, das Problem des Interaktionismus zu wiederholen, nämlich den Prozess der Versicherheitlichung auf Diskurse zu reduzieren. Ein solcher Textualismus ist vor allem von Praxistheorien auf der Basis pragmatistischer Annahmen über die Rolle von nicht-reflektierten, routinierten Handlungsvollzügen kritisiert worden (Reckwitz 2003). Hansens Hinweis auf die Rolle des Körpers lässt sich hier so verstehen, dass materielle Aspekte in die Analyse von Situationen einbezogen werden müssen, um die Genese 
von Bedeutungen auch jenseits kommunikativer Akte nachvollziehen zu können. Sicherheit, so Hansen, kann auch durch den Körper gesprochen werden:

„(...) so that the 'non-security-speech' of Pakistani women might be complemented by the excessive speech of the body. Furthermore, if insecurity can be spoken through the body, it becomes obvious why the body often is a crucial target for those seeking to discipline 'deviant behaviour'" (Hansen 2000: 302).

Erst die Berücksichtigung der Körperlichkeit von Sicherheit erlaubt es die Strategien in den Blick zu nehmen, wie mit Körperlichkeit - und noch allgemeiner - mit Objekten ein Wirkungszusammenhang von Sicherheit generiert wird.

\section{Intersituativität}

Im Folgenden möchte ich nun drei analytische Strategien diskutieren, die auf die Problematik des Provinzialismus und des Textualismus reagieren. Damit soll das Programm eines methodologischen Situationismus in den CSS weiter an Kontur gewinnen. Erstens gilt es, mit Clarke (2005), ein erweitertes Verständnis der Situation zugrunde zu legen. Zweitens soll mit dem Verweis auf das Problem der „Intersituativität" (Hirschauer 2014) eine Perspektive auf die empirische Untersuchung der Mechanismen eröffnet werden, die Situationen miteinander verbinden. Und drittens erlaubt insbesondere die Actor-Network-Theory, die Rolle von Objekten für die Generierung von Bedeutung in der Situationsanalyse zu berücksichtigen.

Mit dem Pragmatismus lässt sich ein theoretisch gehaltvoller Begriff der Situation zum Ausgangspunkt für eine Perspektive in den CSS machen, die der sozialen Konstitution der Wirkungsweise von Sicherheit nachgehen will. Die von Knorr-Cetina rekonstruierte soziologische Tradition des methodologischen Situationismus setzt am pragmatistischen Situationsbegriff an, ohne ihn - wie im Interaktionismus - zwingend auf physische Ko-Präsenz zu beschränken. Aus Sicht der CSS kommen dabei den Techniken der Herstellung von Anwesenheit insofern besondere Bedeutung zu, als Sicherheit häufig mit nicht direkt beobachtbaren, zum Beispiel räumlich und zeitlich entfernten Sachverhalten, entsprechendem antizipatorischen Verhalten und der Imagination zukünftiger Katastrophen, verbunden ist (Anderson 2010; Opitz/Tellmann 2014). Gefahren, Risiken oder Bedrohungen müssen also häufig in Situationen der Sicherheit allererst präsentiert, d.h. situativ zur Anwesenheit gebracht werden. Clarke (2005) hat eine in dieser Hinsicht gewinnbringende Erweiterung des Situationsbegriffs vorgenommen und diesen konsequent einerseits von physischer Ko-Präsenz und andererseits von dem exklusiven Fokus auf zwischenmenschliche Interaktion befreit. Sie knüpft dafür vor allem an Anselm Strauss' Verständnis der Grounded Theory an (Glaser/Strauss 2010) und betont die Komplexität von Situationen, die es zu erfassen gelte. Dafür müsse alles Berücksichtigung finden, was eine Situation ausmacht. Dabei betont sie, dass die Situation stets beides ist - eine raum-zeitliche Lokalisierung von Interaktion und ,the situation of inquiry itself" (Clarke 2005: XXXV). Letzteres macht deutlich, dass Situationen nicht einfach gegeben sind, sondern Forscher*innen sie als solche identifizie- 
ren - freilich nicht beliebig und unabhängig vom empirischen Interaktionsgeschehen, aber doch so, dass das, was als situation of inquiry gilt, in hohem Maße vom Erkenntnisinteresse der Forscher*in abhängt. Diese methodische Herangehensweise entspricht nicht nur Hansens Kritik an der Securitization Theory, sie liefert auch eine Alternative zur Suche nach einer universalen, situationsneutralen Grammatik der Sicherheit. Hansen hat jüngst ihre methodologische Perspektive noch einmal in ihrer Entgegnung auf den Vorwurf der „,methodological whiteness“ (Howell/Richter-Montpetit 2020) der CSS präzisiert:

„(..) the starting point was to grant theoretical recognition to subjects who would aggravate their insecurity if they were speaking. Our discussion of how to theorize this situation must thus start from a recognition of the dangerous situation that those subjects were in and of the need to incorporate the discourses and practices of those who involve themselves politically to address that situation. (...) (I)t was precisely in response to this situation that I made the theoretical move to include bodily practices as an epistemological focus" (Hansen 2020: 5).

Obgleich der Begriff der Situation hier sicherlich nicht in einem theoretisch anspruchsvollen Sinn verwendet wird, geht Hansen doch davon aus, dass die Besonderheit der Situation einen Unterschied mit Blick darauf macht, welche Aspekte für die Entstehung eines Wirkungszusammenhangs der Versicherheitlichung in Betracht gezogen werden müssen. In dieser Hinsicht verfährt Hansen durchaus situationsanalytisch, indem sie zeigt, dass, um die Bedeutung von Sicherheit im Zusammenhang mit Ehrenmorden zu verstehen, auch diejenigen einbezogen werden müssen, die nicht (mehr) sprechen können. Die Abwesenheit des Sprechakts meint situationsanalytisch eben keinesfalls die Abwesenheit von Sicherheit.

Clarkes Vorschlag einer Methodik der Situationsanalyse greift diesen Aspekt in zweifacher Weise auf. Zum einen schlägt sie vor, in die Situationsanalyse alle menschlichen, nicht-menschlichen, diskursiven, historischen, symbolischen oder politischen - Elemente einzubeziehen, die die Situation als eine solche konstituieren (Strübing 2017: 58). Zum anderen schließt Clarke aber auch die Rekonstruktion unterschiedlicher Positionen in die Situationsanalyse ein. Darunter versteht sie ,issues, positions on issues, absences of positions where they might be expected (sites of discursive silence), and differences in discursive positions central to the situation under study" (Clarke 2005: 126). Es geht also um die Erfassung von Positionalitäten in einer Situation, zu der explizit auch Abwesende gehören können, insofern sie als konstitutiv für eine Situation betrachtet werden. Das schließt nicht nur die von Hansen in den Mittelpunkt gerückten Gewaltopfer ein, sondern eben auch alle, ohne die es die Situation, wie wir sie vorfinden, in dieser Form nicht geben würde im vorliegenden Fall beispielsweise Richter, Ehemänner, Unterstützungsnetzwerke aus der Zivilgesellschaft oder Familien. Eine Analyse von Positionalitäten zielt dabei in erster Linie auf die Heterogenität und Konflikthaftigkeit von Positionen in ihren jeweiligen Beziehungen zueinander. Es geht also weniger um die Repräsentation von Positionen als vielmehr um die Rekonstruktion - häufig spannungsgeladener - positionaler Relationen, die Clarke mit einem positional mapping darstellen will. Dabei muss berücksichtigt werden, dass Situationen als soziale Zusammen- 
hänge Relevanzen vorgeben, nach denen soziale Zugehörigkeiten definiert und in eine - oftmals auch hierarchische - Beziehung zueinander gesetzt werden (Hirschauer 2014: 114). Mit anderen Worten: Situationen sind nicht machtfrei. Dies betrifft nicht nur die von der Securitization Theory von Beginn an betonte Autorität von Sprecher*innenpositionen, sondern auch die materiellen Bestandteile einer Situation, etwa die Art und Weise wie sie räumlich strukturiert ist. Mauern, Kameras, aber auch Gerüche, die durch Müll oder Rauch hervorgerufen werden, lassen, wie etwa Ivasiuc (2019) in ihrer Analyse von Lagern für Roma in Italien gezeigt hat, eine Situation entstehen, in der sehr klar zwischen gefährlichen Gruppen im Lager und den gefährdeten außerhalb des Lagers unterschieden wird. Es würde an dieser Stelle zu weit gehen, die methodische Umsetzung eines solchen mappings zu diskutieren. Wichtig erscheint mir vielmehr, dass Clarke hier ein erweitertes Verständnis der Situation entwickelt, das alle konstitutiven Bestandteile der Situation - und dabei insbesondere auch das „silencing von Positionen“ (Strübing 2017: 61) - einbezieht. $^{9}$

Wenn man nicht zuletzt aus Gründen epistemologischer Sparsamkeit (Knorr-Cetina 1988: 22) nicht länger zwischen Kontext und Sprechakt unterscheiden möchte, sondern beides als Bestandteile einer Situation versteht, stellt sich unweigerlich die Frage, wie man sowohl der Situation Vorausgehendes als auch Übersituatives analytisch fassen kann. Situationen weisen offenkundig eine eigene Historizität als auch eine Verbundenheit zu anderen Situationen auf. Die Situation des Ehrenmordes ist mit vorausgehenden und nachfolgenden familiären Situationen oder einer Situation der Rechtsprechung verknüpft. Sie kann nicht stattfinden ohne übersituative Wissensordnungen, die es erlauben, Weiblichkeit auf eine bestimmte Art und Weise zu definieren und die physische Bedrohung durch Familienmitglieder eben nicht als öffentliches Sicherheitsproblem erscheinen zu lassen.

Situationen, in denen Häfen blockiert werden, um das Einlaufen von Rettungsschiffen für Migrant*innen zu blockieren, sind mit Situationen einer diskursiven Versicherheitlichung von Migration in Parlamenten, Medien oder in Alltagsinteraktionen verbunden. Anders gesagt: Eine Analyse der Versicherheitlichung von Migration wird zum einen verschiedene Situationen in den Blick nehmen müssen, in denen Versicherheitlichung jeweils passiert. Und sie sollte zum anderen das Passieren zwischen den Situationen nicht außer Acht lassen, also fragen, auf welche Weise diese Situationen miteinander verbunden sind, wie Bedeutungen von Sicherheit transportiert und übersetzt werden. Man kann Situationen analytisch isolieren, aber zugleich stellt sich damit die empirische Frage, wie Situationen miteinander verbunden sind.

Hirschauer hat in seiner Fortentwicklung des methodologischen Situationismus vorgeschlagen, dies als Frage nach den transsituativen Elementen der Situation und als Frage nach der Intersituativität aufzufassen. Ähnlich haben Susanne Krasmann

9 Methodisch orientiert sich Clarkes Situationsanalyse an den mit der Grounded Theory zusammenhängenden Ansätzen, etwa der „multi-sited ethnography“ (Marcus 1995), schließt aber auch die Diskursanalyse oder visuelle Methoden ein. 
und Christine Hentschel (2019) im Kontext ihrer Analyse des Konzepts der „situational awareness" jüngst dafür plädiert, die Konnektivität von Situationen in den Blick zu nehmen. Situationen sind, so Hirschauer (2014: 112), der hier Erving Goffmans Analyse von Interaktionsordnungen aufgreift, nicht alles. Ihnen gehen größere soziale Felder ebenso voraus wie das transsituative Wissen der Teilnehmenden. Um die der Situation vorausgehenden Aspekte in den Blick zu nehmen, ist es angesichts des analytischen Primats der Situation zielführend, nach den kommunikativen und materiellen Verweisen aus der Situation heraus zu suchen, mit denen sich Situationen selbst transzendieren. Damit rücken die konkreten Verbindungen von Situationen, die in der Situation selbst hergestellt werden, in den Fokus der Situationsanalyse. Hirschauer diskutiert hier vor allem Medien unterschiedlichster Art, mit denen Situationen verbunden werden, etwa symbolisch generalisierte Kommunikationsmedien wie Macht oder Geld, aber auch Bildmedien, mit denen Situationen, wie etwa ein Kriegsgeschehen, in andere Situationen, wie eine Parlamentsdebatte, inkludiert werden müssen. Eine weitere Möglichkeit der Verkettung von Situationen besteht in dem, was Hirschauer die Teleinteraktion nennt, durch die nicht nur Interaktionsteilnehmer*innen, sondern auch ganze Situationen miteinander verbunden werden - wie beispielsweise im Falle militärischer Angriffe mit bemannten Flugzeugen, in denen die Zielsteuerung im Wesentlichen vom Boden aus erfolgt und die Cockpit-Situation mit der der Kommandozentrale zusammengeschaltet ist. ${ }^{10}$ Sowohl für Teleinteraktionen als auch für Medien gilt, dass sie sich als Mechanismen „der steigerbaren Involvierung von Personen in soziale Prozesse“ (Hirschauer 2014: 122) verstehen lassen. Sie vervielfältigen Situationen. In den CSS haben etwa Amicelle et al. (2015) gezeigt, dass security devices wie Scanner, Algorithmen oder Karten eine ähnliche Rolle spielen, indem sie soziale Räume eröffnen und Beziehungen zwischen verschiedenen Kontexten stiften.

Diese Verkettung von Situationen lässt sich nicht nur über Medien erreichen, sondern auch über Objekte, denen die ANT deshalb auch den Status von Akteuren zuspricht, weil sie - ganz im Sinne des Pragmatismus - einen Unterschied im Handeln erwirken. Insbesondere Latour (2005) hat gezeigt, dass Objekte zeitlich und räumlich weit auseinanderliegende Situationen in einen Zusammenhang bringen können. Die Berücksichtigung materieller Objekte in der Situationsanalyse erscheint vor diesem Hintergrund in zweifacher Weise zentral: Zum einen generieren Objekte situative Zusammenhänge, in denen Sicherheit eine spezifische Wirkung entfalten kann. Eine Karte, die Urlaubsorte als gesundheitliche Risikogebiete ausweist, hat für den dort ansässigen Einzelhandel negative ökonomische Konsequenzen. Zum anderen verbindet sie Situationen, nämlich beispielsweise die der familiä-

10 Im Rahmen der ANT hat Walters (2014) unter Rückgriff auf Latours (2001) Konzept der Dingpolitik darüber hinaus gezeigt, wie Objekte, wie beispielsweise Drohnen, Akteure in öffentlichen Kontroversen werden können. Vgl. ähnlich auch Rauer (2014). Ivasiuc (2019) hat am Beispiel der Roma ähnliches für Camps analysiert. In all diesen Arbeiten geht es letztlich darum, die Konstitution von Situationen nicht ausschließlich von den Deutungspraktiken menschlicher Akteur*innen her nachzuvollziehen, sondern gewissermaßen die Dinghaftigkeit von Situationen mit zu berücksichtigen. 
ren Urlaubsplanung mit einem örtlichen Infektionsgeschehen, und produziert auf diese Weise neue Sicherheitspraktiken wie das Testen bei Wiedereinreise.

Intersituativität geht indes nicht in der Analyse der Verkettung ,nachbarschaftlicher" Situationen auf (Hirschauer 2014: 118). Vielmehr gilt es, in der Situationsanalyse vor allem auch der Situation Vorausliegendes und dessen Aktualisierung in den Blick zu nehmen. Dafür fehlt meines Erachtens bislang eine entwickelte analytische Begrifflichkeit. Ich möchte vorschlagen, dafür zum einen an Deweys Beschreibung des Problemlösezyklus anzusetzen und zum anderen - in der Tradition des Pragmatismus - Handeln in Situationen als wissensbasiert zu begreifen. Handeln in Situationen kann demzufolge als alltägliches, sich in Routinen vollziehendes Problemlösen verstanden werden, das ein implizites Wissen der Teilnehmenden aktualisiert. Dafür greifen die Interaktionsteilnehmer*innen auf Heuristiken zurück, die es ihnen erlauben, Situationen zu definieren und in ihnen adäquat zu handeln. Unter Heuristiken lassen sich demzufolge sinnhafte implizite Wissensordnungen, d.h. generalisierte Deutungsmuster, kulturelle Codes und Frames verstehen, auf die Akteur*innen in der Deutung einer Situation - häufig routinemäßig - Bezug nehmen. Heuristiken dienen entsprechend dazu, Komplexität zu reduzieren und situativ handhabbar zu machen. Diese übersituativen Heuristiken werden also in Situationen jeweils aktualisiert und vor allem dann verändert und abgewandelt, wenn sie in der Situation nicht plausibel erscheinen. Dann schalten Akteur*innen gewissermaßen vom Modus des routinehaften Problemlösens in den reflexiven Modus der Reinterpretation einer Situation. Situativ generierte Bedeutungen von Sicherheit gehen in dieser Hinsicht auf übersituative Heuristiken zurück, die es erlauben, Sicherheitsprobleme als solche zu benennen - oder eben, wie im Fall der Ehrenmorde $\mathrm{zu}$ verschweigen und zu individualisieren. Die von Hansen rekonstruierte patriarchale Heuristik, durch die die physische Integrität von Frauen unter bestimmten Umständen nicht als schützenswert erscheint, ermöglicht es, die Sicherheit von Frauen situativ auszuklammern.

Situationen setzen aber Dewey zufolge eben nicht nur implizites Wissen voraus, sondern sie enthalten stets auch eine Handlungskomponente, die mit dem Wissen untrennbar verbunden ist. Problemlösen ist stets beides: Verstehen des Problems und dessen Lösung. Um das übersituative Element der Problemlösung begrifflich zu fassen, bietet es sich an, auf den Begriff des Repertoires zurückzugreifen, wie er unter anderem von Charles Tilly (Della Porta 2013; Klüfers 2014) im Zusammenhang der Protestforschung verwendet wurde. Repertoires sind standardisierte Muster der praktischen Bewältigung von Handlungsproblemen, die Akteur*innen transsituativ zur Verfügung stehen. Auch sie können in bestimmten Situationen als unplausibel erscheinen, so dass neue Praktiken entstehen, die gegebenenfalls selbst zum Repertoire für spätere Situationen werden.

Heuristiken und Repertoires sind dementsprechend Mechanismen der Herstellung von Intersituativität, insofern sie Situationen miteinander verknüpfen. Dies kann auch über Objekte geschehen. Eine Straßensperre etwa gehört zum transsituativen Sicherheitsrepertoire, das in verschiedensten Situationen eingesetzt werden kann. Und zugleich verknüpft es eine Situation der Verhinderung von Mobilität mit 
einer, in der über Maßnahmen der Prävention von Attentaten entschieden wird, und einer, in der Händler*innen ihre Ware auf einen entlegenen Markt bringen wollen. Sicherheit bedeutet in diesen Situationen jeweils unterschiedliches - Blockade, Schutz, Verdienstausfall. Zugleich werden diese Bedeutungen miteinander in eine unmittelbare Beziehung gesetzt. Aus Sicht des methodologischen Situationismus liefe die Fokussierung auf nur eine dieser Bedeutungen auf eine Schrumpfversion von Sicherheit hinaus, die der Komplexität von Sicherheitssituationen nicht gerecht werden würde.

\section{Heuristiken und Repertoires im security-development-nexus}

Heuristiken und Repertoires lassen sich somit als transsituative Mechanismen verstehen, mit denen Sicherheit in jeweils neue Situationen ,übersetzt“ (Stritzel 2011) wird. Eine solche Perspektive erlaubt es nicht zuletzt, die Analyse der Herstellung von Intersituativität um den Aspekt der Historizität von Sicherheit zu erweitern:

,a translational perspective also goes a step further, as the production of security is here examined through notions of process, iteration, travel and evolution/longue durée. This significantly destabilizes any clearcut notions of speaker and audience or any insistence on a single perlocutionary effect. Instead, the notion of translation gives any securitizing move 'deep historicity' by locating the move in a specific temporal and spatial sequence" (Stritzel 2011: 350).

Die Funktionsweise transsituativer Mechanismen - wie Heuristiken und Repertoires - können gut am Beispiel des security-development-nexus veranschaulicht werden. Seit Anfang der 2000er Jahre werden entwicklungspolitische Maßnahmen durch internationale und Entwicklungsorganisationen in einen Zusammenhang mit der Herstellung von Sicherheit gebracht. Entwicklung dient dieser Auffassung zufolge der Herstellung von Sicherheit wie umgekehrt Sicherheit als eine essenzielle Voraussetzung für Entwicklung gilt. Diese „Versicherheitlichung von Entwicklung“ (Brock 2010; Buur, Jensen/Stepputat, 2007; Stern/Öjendal 2010; Brand 2011; Wilkinson 2015) hängt nicht zuletzt mit dem Ziel zusammen, der Entwicklungspolitik eine erhöhte Aufmerksamkeit in der internationalen Politik zu geben und Geberländer ermuntern zu wollen, ihre Ausgaben für entwicklungspolitische Maßnahmen auf das zwischen Industrienationen verabredete Niveau von $0,7 \%$ des Bruttonationaleinkommens anzuheben. Sicherheit, die bis zu diesem Zeitpunkt in Bezug auf damit angesprochene Politikfelder eher als Antipode zu Entwicklung galt, hielt nun Einzug in die Domänen der Entwicklungszusammenarbeit. Umgekehrt galt Entwicklung von da an als wichtiger Bestandteil sicherheitspolitischer, etwa militärischer Interventionen, beispielsweise als begleitende Winning-the-hearts-and-mindsStrategie. Sicherheit erhält damit eine spezifische und gegenüber einem engen Verständnis von Sicherheit deutlich breitere Bedeutung, die auch Maßnahmen der Armuts- und Krankheitsbekämpfung einschließt und deshalb auch nicht zufällig häufig mit dem erweiterten Sicherheitsverständnis des Konzepts der menschlichen Sicherheit in Verbindung gebracht wird (Maclean et al. 2006). 
Kritische Arbeiten zum security-development-nexus haben gezeigt, wie diese neue Bedeutung von Sicherheit als Entwicklung mit einer Heuristik des Paternalismus verbunden ist, die selbst wiederum koloniale Implikationen und Assoziationen besitzt (Duffield 2005). Kennzeichen dieser Heuristik ist ein repräsentatives Sprechen für diejenigen, die selbst nicht sprechen können, dadurch aber auch am Sprechen gehindert werden. Aus historischer Sicht wird hier die Vorstellung der White Man's Burden reaktiviert, der zufolge Verantwortung für diejenigen übernommen werden muss, die sich nicht selbst helfen können, oder die, in den Worten Tony Blairs, „less fortunate than ourselves“ sind (zitiert nach Duffield 2010: 25). Sicherheit wird hier paternalistisch als Schutz für andere verstanden - eine Sichtweise, die erkennbar im liberalen Humanitarismus des 19. Jahrhunderts wurzelt (Barnett 2012).

Vanessa Pupavac (2005) hat diese paternalistische Sicherheitsheuristik am Beispiel der „global therapeutic governance“ veranschaulicht. Dabei vollzieht sie den Relevanzgewinn psychologischer Betrachtungsweisen in der internationalen Entwicklungspolitik nach und kommt zu dem Schluss, dass das Konzept der human security seit den 1990er Jahren dazu geführt hat, die Aufmerksamkeit auf das Wohlergehen (well-being) von Individuen zu richten und damit die ambitionierten Entwicklungsziele aus der Ära modernisierungstheoretischer Ansätze hinter sich zu lassen. Dies führe letztlich zu einem ,anti-development development model“ (Pupavac 2005: 177), das strukturell verursachte massive Unterentwicklung akzeptiere und Programme dafür auflege, Menschen für ihren individuellen Umgang mit Armut zu ertüchtigen. Am Beispiel des Berichts der Weltbank „Voices of the Poor“, der ganz im Sinne einer paternalistischen Heuristik selbst die Stimmen der Armen repräsentiert, arbeitet sie heraus, wie durch den Bezug auf human security Wohlergehen als individuelles und nicht mehr gesellschaftliches Phänomen gedeutet wird. „The report demonstrates how a therapeutic concept of well-being is displacing universal prosperity as the goal of international development policy" (Pupavac 2005: 174).

Eine alternative Heuristik liegt der Situation zugrunde, die Steffen Jensen (2010) in seiner Analyse des Krieges gegen Banden in südafrikanischen Townships in den 1980er Jahren beschreibt. Hier geht es nicht um paternalistischen Schutz, sondern um den Ansatz der Aufstandsbekämpfung, der seit den 1960er Jahren als implizite Wissensordnung für Situationen zur Verfügung steht, in denen der Widerstand gegen eine Herrschaftsordnung beseitigt werden soll. Aufstandsbekämpfung umfasst dabei sowohl Aspekte der Sicherheit als auch der Entwicklung, denn es geht, wie im Vietnam-Krieg erprobt, nicht einfach um die Niederschlagung des Widerstandes, sondern um das Gewinnen der Zustimmung der Bevölkerung. „Hence, counterinsurgency merges development, security and warfare in the most direct manner, with developmental strategies being deployed in the pursuit of state security" (Jensen 2010: 89). Der Krieg in den Townships lässt sich damit als situative Aktualisierung einer Heuristik verstehen, die auf ihre Weise Sicherheit und Entwicklung miteinander verbindet. 
Die Beispiele der therapeutic governance und der Aufstandsbekämpfung zeigen, dass mit Heuristiken, die Sicherheit in den Zusammenhang mit Entwicklung bringen, sehr unterschiedliche Repertoires verbunden sein können. Diese reichen von Entwicklungsmaßnahmen, die der Erhöhung kollektiver Lebensstandards dienen sollen, bis hin zur Anwendung physischer Gewalt. Welche Repertoires situativ aktualisiert werden, hängt nicht zuletzt davon ab, welche in der Situation den Akteur*innen plausibel erscheinen, d.h. auch, welche Akteur*innen Deutungsmacht über die Situation ausüben können. Die Situationsanalyse muss deshalb auch die schon von der Kopenhagener Schule betonte Autorität der Akteur*innen berücksichtigen, obgleich diese nicht allein ausschlaggebend dafür ist, dass sich bestimmte Situationsdefinitionen durchsetzen.

Wie die Beispiele darüber hinaus zeigen, können Heuristiken und Repertoires über längere historische Zeiträume erhalten bleiben. Diese Historizität von Sicherheit kommt nicht zuletzt in der Konzept- und Begriffsgeschichte zum Ausdruck, lässt sich aber mit Hilfe des methodologischen Situationismus auch gewissermaßen realgeschichtlich und gegenwartsbezogen reformulieren. Das Konzept der menschlichen Sicherheit, das für die paternalistische Sicherheitsheuristik von zentraler Bedeutung ist, greift, wie Rothschild (1995) gezeigt hat, auf Vorstellungen der liberalen Aufklärung zurück: „The new political rhetoric of human security in the 1990s is also the old rhetoric of natural or international rights" (Rothschild 1995: 67). Repertoires wie das policing, das essenzieller Teil der Aufstandsbekämpfung in den Townships war, haben ihre eigene Geschichte, die sich auch als eine Geschichte des Wandels von Sicherheitsbedeutungen verstehen lässt. ${ }^{11}$ Eine solche Geschichte müsste zeigen, wie ein situativ zur Anwendung kommendes Repertoire als Mechanismus der Intersituativität gelten kann, der verschiedene Situationen miteinander verbindet und Bedeutungen damit gleichsam über Zeit und Ort stabil hält. Dies ist allerdings keineswegs automatisch der Fall, sondern diese Verbindungen müssen empirisch in der Situation selbst hergestellt und dann von Forscher*innen als solche rekonstruiert werden. Jensen (2010: 88) hat etwa nachzeichnen können, wie in Südafrika das policing in den Townships auf Erfahrungen mit der Art und Weise basierte, wie in den USA in der Stadtteilpolitik gegen Gewalt durch Gangs vorgegangen wurde. Dazu gehörten beispielsweise das Kategorisieren und Kartografieren von bedrohten, fragilen und stabilen Gebieten. Die Geschichte solcher Praktiken kann also von bestimmten Situationen ausgehen, um von dort aus den synchronen und diachronen Verbindungen, d.h. den Kontinuitäten und Diskontinuitäten, nachzuspüren.

11 Ein anderes Beispiel für human security als Sicherheitsheuristik diskutiert Voelkner (2011), die zeigt, wie das Konzept der menschlichen Sicherheit eine situative Verwendung im Zusammenhang mit gesundheitspolitischen policies gegenüber burmesischen Migrant*innen in Thailand findet. 


\section{Zusammenfassung und Ausblick}

Der vorliegende Artikel hat in gewisser Weise einen Weg fortgesetzt, den Jef Huysmans vor über zwanzig Jahren in den CSS beschritten hat, als er vorschlug, Sicherheit als einen ,thick signifier“ zu verstehen (Huysmans 1998). Während Huysmans einen poststrukturalistischen und für die empirisch ausgerichteten CSS wenig anschlussfähigen Ansatz vorlegte, hat dieser Artikel den methodologischen Situationismus als eine interessante Alternative zu den gängigen Ansätzen der CSS ins Spiel gebracht. Damit sind insbesondere drei Absichten verbunden:

Erstens sollte dem insbesondere für die Kopenhagener Schule typischen Fokus auf die Konstruktion von Bedrohungen und ihre politische Wirkung eine Perspektive an die Seite gestellt werden, mit der die demgegenüber analytisch vorgängige Herstellung von Wirkungszusammenhängen in den Blick genommen werden kann, in denen Sicherheit auf vielfältige Weise politisch folgenreich ist. Dies entspricht durchaus dem gestiegenen Interesse an der Performativität von Sicherheit in den CSS (Amicelle et al. 2015; Braun et al. 2019).

Zweitens verfolgte der Beitrag das Ziel, den analytisch unscharfen Begriff des Kontextes, der im Rahmen der soziologischen Wende der Securitization Theory an Prominenz gewann, durch das theoretisch gehaltvollere Konzept der Situation zu ersetzen. Dies ermöglicht zum einen eine präzisere methodische Orientierung in den CSS. Zum anderen geraten damit auch Aspekte der Konstruktion von Sicherheit jenseits des Sprechaktes in den Blick, etwa Objekte, aber auch die Rolle impliziter Wissensordnungen oder standardisierter und routinierter Problemlösungen. Diese sind selbstverständlich von den CSS schon seit einiger Zeit gerade auch in Abgrenzung von der Sprechakt-Fokussierung der Kopenhagener Schule untersucht worden (vgl. u.a. Ceyhan 2002; Aradau 2010; Rothe 2016; de Goede 2018).

Deshalb bestand ein drittes Anliegen des Artikels darin, für solche Forschungen innerhalb der CSS einen gemeinsamen analytischen Rahmen zu entwickeln. Am Beispiel der Analyse des silencing im Kontext von Ehrenmorden und der Forschungen zum security-development-nexus wurde sukzessive die Perspektive des methodologischen Situationismus entfaltet, die an ein pragmatistisches Verständnis der Konstruktion von Bedeutungen anschließt, dieses dann aber um ein erweitertes Verständnis der Situation und das Konzept der Intersituativität ergänzt. Eine solche methodologische Perspektive richtet ihr Augenmerk dementsprechend auch weniger auf die strukturierenden Erfolgsbedingungen von Versicherheitlichung, sondern fragt nach den Herstellungsweisen solcher sicherheitsrelevanter Situationen, zu denen vor allem auch transsituative Elemente gehören. Eine Analyse von situationsübergreifenden, aber jeweils situativ aktualisierten Heuristiken und Repertoires ,reveals how new conceptualizations of security at once play on the established meaning of security and invest it with new meaning" (Philipsen 2020).

Mit dem Vorschlag einer situationsanalytischen Reformulierung der kontextualistischen Variante der Securitization Theory ist natürlich die Frage verbunden, wieviel von den zentralen Annahmen der Securitization Theory übrigbleibt und ob hier nicht der sprechakttheoretische Boden der Versicherheitlichungstheorie endgültig 
zugunsten des Pragmatismus verlassen wird. Umgekehrt ließe sich argumentieren, dass insbesondere die Arbeiten von Bigo und der an ihn anschließenden Internationalen Politischen Soziologie schon längst gezeigt haben, dass die sprechakttheoretische Fundierung in eine Engführung des Verständnisses von Versicherheitlichung mündet, so dass sich auch eine situationsanalytische Reformulierung aus dieser Perspektive erübrigt. Mir ging es in diesem Beitrag darum, beiden Argumenten gegenüber eine Mittelposition einzunehmen, indem die situationsanalytische Reformulierung darauf zielt, den Konstitutionszusammenhang von Versicherheitlichung als solchen zu erfassen. $\mathrm{Zu}$ ihm mögen Sprechakte und durch sie hervorgebrachte Bedrohungskonstruktionen ebenso gehören wie alltägliche Routinen sicherheitsbezogenen Handelns und materielle Artefakte. Wie diese aber in einen empirischen Zusammenhang gebracht werden und wie weitere - typischerweise als kontextuelle Faktoren bezeichnete - Elemente wie etwa die Interpretationshorizonte eines Publikums in die Konstruktion von Sicherheit hineinspielen, lässt sich bislang analytisch nicht hinreichend fassen. Insofern zielt der Beitrag auch nicht auf eine Ersetzung theoretischer Perspektiven der CSS, sondern auf das Potenzial ihrer methodologischen Verknüpfung. Damit einher geht vor allem auch ein Plädoyer, Theorien in den CSS stärker als in der Kopenhagener Schule empirisch zu generieren, indem etwa empirisch danach gefragt wird, wie bestimmte Deutungsmuster dazu führen, bestimmte Situationen - beispielsweise durch historische Vergleiche - miteinander zu verbinden und Referenzobjekte darüber zu versicherheitlichen.

Damit richtet sich das Interesse dann vor allem auch auf eine - nicht zuletzt historisch ausgerichtete - CSS, die nach den Kontinuitäten und Diskontinuitäten von Sicherheitskonstruktionen fragt und sich dem Umstand bewusst ist, dass „Sicherheit (...) eine zutiefst historische Kategorie (ist)“ (Conze 2009: 17). Daase hat schon vor einiger Zeit auf die Fruchtbarkeit einer stärker historisch ausgerichteten CSS hingewiesen, durch die "Sozialwissenschaften und Geschichtswissenschaft (...) an analytischer Aussagekraft gewinnen" (Daase 2012: 405). Obgleich der methodologische Situationismus auf den ersten Blick eine starke Gegenwartsorientierung aufweist, eignet er sich dennoch für eine solche stärker an der Historisierung von Sicherheit interessierten Ausrichtung der CSS: Schon der Pragmatismus hat hervorgehoben, dass Praktiken immer schon historisch eingebettet sind und sich Vergangenes in der Situation aktualisiert und materialisiert. Sicherheit wird nicht zuletzt immer vor dem Hintergrund historischer Erfahrungen und mit Rückgriff auf überlieferte Bedeutungen definiert. Dies aufgreifend betont die Situationsanalyse im Rahmen der CSS in besonderem Maße die Notwendigkeit, nicht nur - analog zur Begriffs- und Konzeptgeschichte - die Historizität von Sicherheit zu berücksichtigen, sondern auch den Wandel ihrer Wissensordnungen und Praktiken in den Mittelpunkt zu rücken. Freilich bleibt eine solche Historisierung hier an die Prämissen des methodologischen Situationismus gebunden, denen zufolge „history itself appears as a succession of microsituations chained together by a variety of interrelations" (Knorr-Cetina 1988: 36). Und obgleich sich eine solche historisch interessierte Situationsanalyse auf eine lange soziologische Tradition berufen kann, steht ihre Rezeption in den CSS erst am Anfang. 


\section{Literatur}

Adler, Emanuel/Pouliot, Vincent 2011: International Practices, in: Adler, Emanuel/Pouliot, Vincent (Hrsg.): International Practices, Cambridge, 3-35.

Amicelle, Anthony/Aradau, Claudia/Jeandesboz, Julien 2015: Questioning Security Devices: Performativity, Resistance, Politics, in: Security Dialogue 46: 4, 293-306.

Amoore, Louise 2013: The Politics of Possibility: Risk and Security beyond Probability, Durham.

Anderson, Ben 2010: Preemption, Precaution, Preparedness: Anticipatory Action and Future Geographies, in: Progress in Human Geography 34: 6, 777-798.

Aradau, Claudia 2010: Security that Matters: Critical Infrastructure and Objects of Protection, in: Security Dialogue 41: 5, 491-514.

Baldwin, David A. 1997: The Concept of Security, in: Review of International Studies 23: 1, 5-26.

Balzacq, Thierry 2002: Security, Identity, and Symbolic Interactionism, in: International Review of Sociology 12: 3, 469-506.

Balzacq, Thierry 2005: The Three Faces of Securitization: Political Agency, Audience and Context, in: European Journal of International Relations 11: 2, 171-201.

Balzacq, Thierry 2011: Securitization Theory: How Security Problems Emerge and Dissolve, London.

Balzacq, Thierry 2015: Contesting Security: Strategies and Logics (PRIO new security studies), London.

Barnett, Michael N. 2012: International Paternalism and Humanitarian Governance, in: Global Constitutionalism 1: 3, 485-521.

Bellanova, Rocco/Jacobsen, Katja Lindskov/ Monsees, Linda 2020: Taking the Trouble: Science, Technology and Security Studies, in: Critical Studies on Security, 8:2, 87-100.

Bertrand, Sarah 2018: Can the Subaltern Securitize? Postcolonial Perspectives on Securitization Theory and Its Critics, in: European Journal of International Security 3: 03, 281-299.

Bigo, Didier 2008: Globalized (in)Security. The Field and the Ban-Opticon, in: Tsoukala, Anastassia/Bigo, Didier (Hrsg.): Terror, Insecurity and Liberty: Illiberal Practices of Liberal Regimes after 9/11, London, 10-48.

Bilgin, Pinar 2010: The 'Western-Centrism' of Security Studies: 'Blind Spot' or Constitutive Practice?, in: Security Dialogue 41: 6, 615-622.

Bilgin, Pinar 2011: The Politics of Studying Securitization? The Copenhagen School in Turkey, in: Security Dialogue 42: 4-5, 399-412.

Blumer, Herbert 1986: Symbolic Interactionism: Perspective and Method, Berkeley.

Booth, Ken 2011: Critical Security Studies, in: Christie, Daniel J. (Hrsg.): The Encyclopedia of Peace Psychology, Oxford, in: http://doi.wiley.com/10.1002/9780470672532. wbepp072; 21.8.2020.

Brand, Alexander 2011: Sicherheit über alles? Die schleichende Versicherheitlichung deutscher Entwicklungspolitik, in: PERIPHERIE - Politik • Ökonomie • Kultur 31: 122-123, 209-235.

Brock, Lothar 2010: The Securitization of Development. More Political Leverage for Africa or More Interference from the Outside?, in: Tagou, Celestin (Hrsg.): The Dynamics of Conflict, Peace and Development in African Societies, Yaoundé, 269-280.

Bubandt, Nils 2005: Vernacular Security: The Politics of Feeling Safe in Global, National and Local Worlds, in: Security Dialogue 36: 3, 275-296.

Bueger, Christian 2018: Security as Practice, in: Dunn Cavelty, Myriam/Balzacq, Thierry (Hrsg.): Routledge Handbook of Security Studies, 126-135.

Bueger, Christian/Gadinger, Frank 2008: Praktisch gedacht! Praxistheoretischer Konstruktivismus in den Internationalen Beziehungen, in: Zeitschrift für Internationale Beziehungen 15: 2, 273-302. 
Braun, Benjamin/Schindler, Sebastian/Wille, Tobias 2019: Rethinking Agency in International Relations: Performativity, Performances and Actor-Networks, in: Journal of International Relations and Development 22: 4, 787-807.

Burgess, J. Peter 2017: Security Studies, in: Gauillaume, Xavier/Bilgin, Pinar (Hrsg.): Routledge Handbook of International Political Sociology, London/New York, 233-244.

Butler, Judith 1993: Bodies That Matter: On the discursive limits of "sex", Abingdon.

Buur, Lars (Hrsg.) 2007: The Security-Development Nexus: Expressions of Sovereignty and Securitization in Southern Africa, Uppsala.

Buzan, Barry 1983: People, States, and Fear: the National Security Problem in International Relations, Chapel Hill, NC.

Buzan, Barry/Hansen, Lene 2009: The Evolution of International Security Studies, Cambridge.

Buzan, Barry/Waver, Ole/Wilde, Jaap de 1998: Security: A New Framework for Analysis, Boulder, CO.

Campbell, David 1992: Writing Security: United States Foreign Policy and the Politics of Identity, Minneapolis, MN.

Ceyhan, Ayse 2002: Technologization of Security: Management of Uncertainty and Risk in the Age of Biometrics, in: Surveillance \& Society 5: 2, in: https://ojs.library.queensu.ca/ ind ex.php/surveillance-and-society/article/view/3430; 22.8.2020.

Ciută, Felix 2009: Security and the Problem of Context: A Hermeneutical Critique of Securitisation Theory, in: Review of International Studies 35: 2, 301-326.

Clarke, Adele E. 2005: Situational Analysis: Grounded Theory after the Postmodern Turn, Thousand Oaks, CA.

Clarke, Adele E./Friese, Carrie/Washburn, Rachel (Hrsg.) 2015: Situational Analysis in Practice: Mapping Research with Grounded Theory, Walnut Creek, CA.

Conze, Eckart 2009: Die Suche nach Sicherheit: eine Geschichte der Bundesrepublik Deutschland von 1949 bis in die Gegenwart, 1. Aufl., München.

Conze, Werner 1984: Art. Sicherheit, Schutz, in: Brunner, Otto/Conze, Werner/Koselleck, Reinhart (Hrsg.): Geschichtliche Grundbegriffe. Historisches Lexikon zur politisch-sozialen Sprache in Deutschland. Bd. 5, Stuttgart, 831-862.

Côté, Adam 2016: Agents without Agency: Assessing the Role of the Audience in Securitization Theory, in: Security Dialogue 47: 6, 541-558.

Daase, Christopher 2010: Der Wandel der Sicherheitskultur - Ursachen und Folgen des erweiterten Sicherheitsbegriffs, in: Zoche, Peter/Kaufmann, Stefan/Haverkamp, Rita (Hrsg.): Zivile Sicherheit: gesellschaftliche Dimensionen gegenwärtiger Sicherheitspolitiken, Bielefeld, 139-158.

Daase, Christopher 2012: Die Historisierung der Sicherheit. Anmerkungen zur historischen Sicherheitsforschung aus politikwissenschaftlicher Sicht, in: Geschichte und Gesellschaft 38: 3, 387-405.

de Goede, Marieke 2018: The Chain of Security, in: Review of International Studies 44: 1, 24-42.

Della Porta, Donatella 2013: Repertoires of Contention, in: Snow, David A/Della Porta, Donatella/Klandermans, Bert/McAdam, Doug (Hrsg.): The Wiley-Blackwell Encyclopedia of Social and Political Movements, Chichester.

Der Derian, James 1995: The Value of Security: Hobbes, Marx, Nietzsche, and Baudrillard, in: Lipschutz, Ronnie (Hrsg.): On Security, Band New Directions in world politics, New York, NY, 24-45.

Derrida, Jacques 1990: Die différance, in: Engelmann, Peter (Hrsg.): Postmoderne und Dekonstruktion. Texte philosophischer Autoren der Gegenwart., Stuttgart, 76-111.

Dewey, John 1938: Logic: The Theory of Inquiry, New York, NY.

Dillon, Michael/Neal, Andrew W. (Hrsg.) 2008: Foucault on Politics, Security and War, New York, NY. 
Duffield, Mark 2005: Getting Savages to Fight Barbarians: Development, Security and the Colonial Present: Analysis, in: Conflict, Security \& Development 5: 2, 141-159.

Duffield, Mark 2010: The Development-Security Nexus in Historical Perspective: Governing the World of Peoples, in: Sorensen, Jens Stilhoff (Hrsg.): Challenging the Aid Paradigm: Western Currents and Asian Alternatives, Basingstoke, 25-46, in: http://link.springer.co $\mathrm{m} / 10.1057 / 9780230277281 \_2 ; 22.8 .2020$.

Gallie, William B. 1955: Essentially Contested Concepts, in: Proceedings of the Aristotelian Society 56: 1, 167-198.

Glaser, Barney G./Strauss, Anselm L./Paul, Axel T. 2010: Grounded theory: Strategien qualitativer Forschung, 3., unveränderte Auflage, Bern.

Goffman, Erving 1972: The Neglected Situation, in: Giglioli, P P (Hrsg.): Language and Social Context, Harmondsworth, 61-66.

Guzzini, Stefano 2015: A Dual History of „Securitisation“, Copenhagen, in: http://pure.diis.dk /ws/files/218242/DIIS_WP_2015_02_A_dual_history_of_Securitisation.pdf; 29.6.2016.

Hansen, Lene 2000: The Little Mermaid's Silent Security Dilemma and the Absence of Gender in the Copenhagen School, in: Millennium: Journal of International Studies 29: 2, 285-306.

Hansen, Lene 2020: Are 'Core' Feminist Critiques of Securitization Theory Racist? A Reply to Alison Howell and Melanie Richter-Montpetit, in: Security Dialogue 51: 4, 378-385.

Hellmann, Gunther 2017: Pragmatismus in den Internationalen Beziehungen, in: Sauer, Frank/Masala, Carlo (Hrsg.): Handbuch Internationale Beziehungen, Wiesbaden, 359-397.

Hirschauer, Stefan 2014: Intersituativität. Teleinteraktionen und Koaktivitäten jenseits von Mikro und Makro, in: Zeitschrift für Soziologie, Sonderheft Interaktion - Organisation Gesellschaft revisited: 109-133.

Howell, Alison/Richter-Montpetit, Melanie 2020: Is Securitization Theory Racist? Civilizationism, Methodological Whiteness, and Antiblack Thought in the Copenhagen School, in: Security Dialogue 51: 1, 3-22.

Huysmans, Jef 1998: Security! What Do You Mean?: From Concept to Thick Signifier, in: European Journal of International Relations 4: 2, 226-255.

Huysmans, Jef 2011: What's in an Act? On Security Speech Acts and Little Security Nothings, in: Security Dialogue 42: 4-5, 371-383.

Ivasiuc, Ana 2019: Reassembling Insecurity: The Power of Materiality, in: Kreide, Regina/ Langenohl, Andreas (Hrsg.): Conceptualizing Power in Dynamics of Securitization, Baden-Baden, 367-394.

Jensen, Steffen 2010: The Security and Development Nexus in Cape Town: War on Gangs, Counterinsurgency and Citizenship, in: Security Dialogue 41: 1, 77-97.

Kampmann, Christoph/Carl, Horst 2021: Historische Sicherheitsforschung und die Sicherheit des Friedens, in: Dingel, Irene et al (Hrsg.): Handbuch Frieden im Europa der Frühen Neuzeit / Handbook of Peace in Early Modern Europe, Berlin 2021, 529-550.

Katzenstein, Peter J. (Hrsg.) 1996: The Culture of National Security: Norms and Identity in World Politics (New directions in world politics), New York, NY.

Kaufmann, Franz-Xaver 1973: Sicherheit als soziologisches und sozialpolitisches Problem. Untersuchungen zu einer Wertidee hochdifferenzierter Gesellschaften, 2. Auflage, Stuttgart.

Krasmann, Susanne/Hentschel, Christine 2019: "Situational Awaremess": Rethinking Security in Times of Urban Terrorism, in: Security Dialogue 50: 2, 181-197.

Ketzmerick, Maria 2019: Staat, Sicherheit und Gewalt in Kamerun: Postkoloniale Perspektiven auf den Dekolonisierungsprozess unter französischer UN-Treuhandverwaltung, in: https://doi.org/10.14361/9783839449042; 21.8.2020.

Klüfers, Philipp 2014: Security Repertoires: Towards a Sociopragmatist Framing of Securitization Processes, in: Critical Studies on Security 2: 3, 278-292. 
Knorr-Cetina, Karin 1988: The Micro-social Order. Towards a Reconception, in: Fielding, Nigel (Hrsg.): Actions and Structure: Research Methods and Social Theory, London, 21-53.

Langenohl, Andreas 2019: Dynamics of Power in Securitization: Towards a Relational Understanding, in: Kreide, Regina/Langenohl, Andreas (Hrsg.): Conceptualizing Power in Dynamics of Securitization, Baden-Baden, 23-66.

Langenohl, Andreas 2020: Articulating Sovereignty within the Infrastructural Imagination: The Case of the Securitisation of Finance as 'Critical Infrastructure', in: Politikon 47: 1, 4-23.

Latour, Bruno 2001: Eine Soziologie ohne Objekt?: Anmerkungen zur Interobjektivität, in: Berliner Journal für Soziologie 11: 2, 237-252.

Latour, Bruno 2005: Reassembling the Social: an Introduction to Actor-Network-Theory (Clarendon Lectures in Management Studies), Oxford.

Lemay-Hébert, Nicolas 2018: Living in the Yellow Zone: The Political Geography of Intervention in Haiti, in: Political Geography 67: 88-99.

Léonard, Sarah/Kaunert, Christian 2011: Reconceptualizing the Audience in Securitization Theory, in: Balzacq, Thierry (Hrsg.): Securitization theory: how security problems emerge and dissolve, Milton Park, Abingdon, Oxfordshire 57-76.

Lipschutz, Ronnie D. 1995: On Security, in: Lipschutz, Ronnie D. (Hrsg.): On Security, New York, NY, 1-23.

Luhmann, Niklas 1990: Identität - was oder wie?, in: Soziologische Aufklärung 5 Konstruktivistische Perspektiven, Opladen, 14-30.

Petersen, Karen Lund 2013: The Corporate Security Professional: A Hybrid Agent between Corporate and National Security, in: Security Journal 26: 3, 222-235.

MacLean, Sandra J./Black, David R./Shaw, Timothy M. 2006: Introduction: A Decade of Human Security: What Prospects for Global Governance and New Multilateralism, in: A Decade of Human Security: Global Governance and New Multilateralisms., Farnham, 3-18.

Marcus, George E. 1995: Ethnography in/of the World System: The Emergence of Multi-Sited Ethnography, in: Annual Review of Anthropology 24: 1, 95-117.

McDonald, Matt 2008: Securitization and the Construction of Security, in: European Journal of International Relations 14: 4, 563-587.

Mead, George Herbert 1968: Geist, Identität und Gesellschaft: aus der Sicht des Sozialbehaviorismus (Suhrkamp Taschenbuch Wissenschaft, Band 28), Frankfurt am Main.

Neocleous, Mark 2006: From Social to National Security: On the Fabrication of Economic Order, in: Security Dialogue 37: 3, 363-384.

Opitz, Sven 2017: Simulating the World: The Digital Enactment of Pandemics as a Mode of Global Self-Observation, in: European Journal of Social Theory 20: 3, 392-416.

Opitz, Sven/Tellmann, Ute 2015: Future Emergencies: Temporal Politics in Law and Economy, in: Theory, Culture \& Society 32: 2, 107-129.

Peoples, Columba/Vaughan-Williams, Nick 2010: Critical Security Studies: An Introduction, Milton Park, Abingdon, Oxon; New York, NY.

Pettenkofer, Andreas 2017: Beweissituationen. Zur Rekonstruktion des Konzepts sozialer Praktiken, in: Dietz, Hella/Nungesser, Frithjof/Pettenkofer, Andreas (Hrsg.): Pragmatismus und Theorien sozialer Praktiken: vom Nutzen einer Theoriedifferenz, Frankfurt New York, 119-160.

Philipsen, Lise 2020: Performative Securitization: From Conditions of Success to Conditions of Possibility, in: Journal of International Relations and Development 23: 1, 139-163.

Pupavac, Vanessa 2005: Human Security and the Rise of Global Therapeutic Governance: Analysis, in: Conflict, Security \& Development 5: 2, 161-181.

Rauer, Valentin 2014: Inter-Objektivität. Sicherheitskultur aus Sicht der Akteur-NetzwerkTheorie, in: Daase, Christopher/Offermann, Philipp/Rauer, Valentin (Hrsg.): Sicherheitskultur. Soziale und politische Praktiken der Gefahrenabwehr, 33-56. 
Reckwitz, Andreas 2003: Grundelemente einer Theorie sozialer Praktiken: Eine sozialtheoretische Perspektive, in: Zeitschrift für Soziologie 32: 4, 282-301.

Robinson, Fiona 2011: The Ethics of Care. A Feminist Approach to Human Security, Philadelphia

Rothe, Delf 2016: Securitizing Global Warming: a Climate of Complexity (Routledge Studies in Resilience), London.

Rothschild, Emma 1995: What Is Security?, in: Daedalus 124: 3, 53-98.

Salter, Mark 2008: Securitization and Desecuritization: A Dramaturgical Analysis of the Canadian Air Transport Security Authority, in: Journal of International Relations and Development 11: 4, 321-349.

Schegloff, Emanuel A. 1997: Whose Text? Whose Context?, in: Discourse \& Society 8: 2, 165-187.

Schlegel, Steve/Schuck, Christoph 2017: Denn nur vom Nutzen wird die Welt regiert? Zum abnehmenden Stellenwert der Critical Security Studies/Welsh School in den IB, in: Zeitschrift für Internationale Beziehungen 24: 1, 100-126.

Schlichte, Klaus/Veit, Alex 2010: Drei Arenen: Warum Staatsbildung von außen so schwierig ist, in: Bonacker, Thorsten/Daxner, Michael/Free, Jan H./Zürcher, Christoph (Hrsg.): Interventionskultur: Zur Soziologie von Interventionsgesellschaften, Wiesbaden, in: http:// link.springer.com/10.1007/978-3-531-92219-5; 22.8.2020.

Schütz, Alfred/Luckmann, Thomas 1979: Strukturen der Lebenswelt, Frankfurt am Main.

Stern, Maria/Öjendal, Joakim 2010: Mapping the Security-Development Nexus: Conflict, Complexity, Cacophony, Convergence?, in: Security Dialogue 41: 1, 5-29.

Stritzel, Holger 2007: Towards a Theory of Securitization: Copenhagen and Beyond, in: European Journal of International Relations 13: 3, 357-383.

Stritzel, Holger 2011: Security, the Translation, in: Security Dialogue 42: 4-5, 343-355.

Stritzel, Holger 2014: Security in Translation: Securitization Theory and the Localization of Threat (New Security Challenges Series), Basingstoke.

Strübing, Jörg 2017: Where is the Meat/d? Pragmatismus und Praxistheorien als reziprokes Ergänzungsverhältnis, in: Dietz, Hella/Nungesser, Frithjof/Pettenkofer, Andreas (Hrsg.): Pragmatismus und Theorien sozialer Praktiken: vom Nutzen einer Theoriedifferenz, Frankfurt, 41-75.

Tickner, J. Ann 2004: Feminist Responses to International Security Studies, in: Peace Review 16: 1, 43-48.

Voelkner, Nadine 2011: Managing Pathogenic Circulation: Human Security and the Migrant Health Assemblage in Thailand, in: Security Dialogue 42: 3, 239-259.

von Lucke, Franziskus/Diez, Thomas/Wellmann, Zehra 2016: Klimakämpfe: Eine komparative Studie der Versicherheitlichung von Klimawandel, in: ZIB Zeitschrift für Internationale Beziehungen 23: 2, 112-143.

Vuori, Juha A. 2008: Illocutionary Logic and Strands of Securitization: Applying the Theory of Securitization to the Study of Non-Democratic Political Orders, in: European Journal of International Relations 14: 1, 65-99.

Wcever, Ole 1995: Securitization and Desecuritization, in: Lipschutz, Ronnie D. (Hrsg.): On Security, New York, 46-86.

Weever, Ole 1996: European Security Identities, in: Journal of Common Market Studies 34: 1, 103-132.

Wcever, Ole 2000: The EU as a Security Actor: Reflections from a Pessimistic Constructivist on Post-Sovereign "Security Orders", in: Kelstrup, Morten/Williams, Michael C. (Hrsg.): International Relations Theory and the Politics of European Integration, London, 250-294.

Waver, Ole 2008: Peace and Security: Two Evolving Concepts and Their Changing Relationship, in: Brauch, Hans Günter/Spring, Úrsula Oswald/Mesjasz, Czeslaw/Grin, John/ Dunay, Pál/Behera, Navnita Chadha/Chourou, Béchir/Kameri-Mbote, Patricia/Liotta, P. $H$. (Hrsg.): Globalization and Environmental Challenges: Reconceptualizing Security in 
the 21st Century, Band 3, Berlin, 99-111, in: http://link.springer.com/10.1007/978-3-540 $-75977-5 ; 22.8 .2020$.

Wever, Ole/Buzan, Barry 2020: Racism and Responsibility - The Critical Limits of Deepfake Methodology in Security Studies: A Reply to Howell and Richter-Montpetit, in: Security Dialogue 51: 4, 386-394.

Walters, William 2014: Drone Strikes, Dingpolitik and beyond: Furthering the Debate on Materiality and Security, in: Security Dialogue 45: 2, 101-118.

Waltz, Kenneth N. 1959: Man, the State, and War: a Theoretical Analysis, New York, NY.

Weldes, Jutta/Laffey, Mark/Gusterson, Hugh/Duvall, Raymond 1999: Introduction: Constructing Insecurity, in: Weldes, Jutta/Laffey, Mark/Gusterson, Hugh/Duvall, Raymond (Hrsg.): Cultures of Insecurity: States, Communities, and the Production of Danger, Minneapolis, MN, 1-34.

Wilkinson, Cai 2015: The Securitization of Development, in: Jackson, Paul (Hrsg.): Handbook of International Security and Development, 32-46.

Wilkinson, Claire 2007: The Copenhagen School on Tour in Kyrgyzstan: Is Securitization Theory Useable Outside Europe?, in: Security Dialogue 38: 1, 5-25. 